\title{
Quasi-local mass functionals and generalized inverse mean curvature flow
}

\author{
JefFrey D. StREets
}

\begin{abstract}
Let $M$ be an asymptotically flat 3-manifold with non-negative scalar curvature. In [H. Bray, A family of quasi-local mass functionals with monotone flows, Proceedings of the International Congress of Mathematical Physics, Lisbon, 2003.] Hubert Bray defines a family of quasi-local mass functionals which are monotone for surfaces smoothly satisfying a certain generalization of inverse mean curvature flow in $M$. We show that a weak solution in the sense of Huisken-Ilmanen [G. Huisken and T. Ilmanen, The inverse mean curvature flow and the Riemannian Penrose inequality, J. Differen. Geom. 59, 2001, 353-437.] exists for a wide class of flows including these with monotone quasi-local mass functionals, and we show that the monotonicity holds for the weak flow as well. As shown in [H. Bray, A family of quasi-local mass functionals with monotone flows, Proceedings of the International Congress of Mathematical Physics, Lisbon, 2003.], a Penrose-type inequality for connected surfaces is an immediate corollary.
\end{abstract}

\section{Introduction}

In this paper we will consider a certain generalization of the inverse mean curvature flow. Specifically, let $\left(M^{n}, g\right)$ be a smooth Riemannian manifold, and let $x: N \times[0, T] \rightarrow M$ be a smooth family of hypersurfaces satisfying

$$
\frac{\partial x}{\partial t}=\frac{\nu}{f(H)}
$$

where $H$ is the mean curvature of $N_{t}$ at the point $x, \nu$ is the outward unit normal, $\frac{\partial x}{\partial t}$ is the normal velocity field along the surface $N_{t}$ and $f$ is a function $f: \mathbb{R} \rightarrow \mathbb{R}$. There are two specific geometric applications motivating the study of flows of this type, both arising from general relativity. First, restrict to the case where $\left(M^{3}, g\right)$ is an asymptotically flat, time symmetric space-like slice of a spacetime with $R_{g} \geq 0$, a consequence of the dominant energy condition for the spacetime. In [4], Bray defines a one-parameter 
family of quasi-local mass functionals for $\Sigma$ a smooth surface in $\left(M^{3}, g\right)$. Specifically for any $c \in[0, \infty)$ we define

$$
m_{c}(\Sigma)=\frac{\sqrt{A}}{16 \pi}\left(1-\frac{1}{16 \pi} \int_{\Sigma} \frac{1}{c^{2}} F(c H)\right)-c
$$

where $y=F(x)$ is the implicit solution to

$$
x^{2}=y+y^{3 / 2} .
$$

Bray showed that this mass functional is monotonic when smoothly flowing the surface $\Sigma$ in the outward normal direction with speed

$$
\eta_{c}=\frac{2 c}{f(c H)}
$$

where $f=F^{\prime}$. Bray further conjectured that this flow exists globally in a weak sense defined by Huisken and Ilmanen in [8], and that the asymptotic limit of (1.2) is the usual ADM mass. This would show $m_{\mathrm{ADM}} \geq m_{c}^{F}(\Sigma)$. After taking the supremum over different choices of $c$ we then conclude the following theorem.

Theorem 1.1. Let $(M, g)$ be a complete connected asymptotically flat 3manifold with non-negative scalar curvature. Say the boundary of $M$ is a connected, compact, outer minimizing surface $\Sigma$. Then

$$
m_{\mathrm{ADM}} \geq m_{H}(\Sigma)+\frac{|\Sigma|^{1 / 2}}{(16 \pi)^{3 / 2}} \cdot \frac{\max \left(0, \int_{\Sigma} H^{3}-(16 \pi)^{3 / 2} /|\Sigma|^{1 / 2}\right)^{2}}{6 \int_{\Sigma} H^{4}} .
$$

A second geometric application comes from the study of negative point mass singularities, which were introduced by Bray in [5] and explored further in [9]. Bray takes a negative point mass Schwarzchild metric

$$
d s^{2}=\left(1+\frac{m}{2|x|}\right)^{4} d \vec{x}^{2}-\left(\frac{1-\frac{m}{2|x|}}{1+\frac{m}{2|x|}}\right)^{2} d t^{2}
$$

where $m<0$ is a starting point and proceeds to develop a theory of more general negative point mass singularities. This analysis suggests that the 
quasi-local mass functional

$$
-\left(\frac{1}{16 \pi} \int_{\Sigma} H^{4 / 3} d A\right)^{3 / 2}
$$

could take the place of the Hawking mass for negative point mass singularities. As it turns out $\frac{t}{3}-\frac{1}{16 \pi} \int_{\Sigma} H^{4 / 3}$ is monotonic under Equation (1.1) with $f(x)=x^{1 / 3}$. It is as yet unclear precisely what quasi-local mass functionals are appropriate for the study of these singularities, but solutions to these generalized flows may prove important. We take up this question in further detail in [10].

We return for now to the case of a general function $f$ in (1.1). In the level set formulation of this flow the evolving surfaces will be given as the level sets of a function $u$ by:

$$
N_{t}=\partial\{x \mid u(x)<t\}
$$

where now $u$ satisfies:

$$
f\left(\operatorname{div}_{M} \frac{\nabla u}{|\nabla u|}\right)=|\nabla u| .
$$

Example 1.2. If $f(x)=x^{\frac{1}{\alpha}}$ for $\alpha \neq 1$, then we have to solve the equation:

$$
\operatorname{div}_{M} \frac{\nabla u}{|\nabla u|}=|\nabla u|^{\alpha} \text {. }
$$

In the case of $\mathbb{R}^{n}-\{0\}$ we see that the function

$$
u(x)=\frac{\alpha(n-1)}{\alpha-1}|x|^{\frac{\alpha-1}{\alpha}}
$$

is a solution corresponding to an expanding sphere.

Proposition 1.3. Equation (1.7) is elliptic for $H \geq 0$ if and only if $f^{\prime}(x)>$ 0 for $x \geq 0$.

Proof. We must compute the linearization of Equation (1.7). So, let $\left\{u_{s}\right\}$ be a family of functions such that $u_{0}=u$ and $\frac{\partial}{\partial s} u_{s \mid s=0}=v$. Then

$$
\begin{aligned}
\frac{\partial}{\partial s}\left(f\left(\operatorname{div}_{M} \frac{\nabla u_{s}}{\left|\nabla u_{s}\right|}\right)-\left|\nabla u_{s}\right|\right) & =f^{\prime}(H) \frac{\partial}{\partial s} \operatorname{div}_{M} \frac{\nabla u_{s}}{\left|\nabla u_{s}\right|}-\frac{\partial}{\partial s}\left|\nabla u_{s}\right| \\
& =f^{\prime}(H) \operatorname{div}_{M} \frac{\nabla v}{|\nabla u|}+\text { l.o.t. }
\end{aligned}
$$

Thus as $|\nabla u|$ is generally not zero, the result follows. 
Thus we see our first condition on $f$ which will be necessary for solutions to generally exist. In the course of developing solutions to this generalized flow, we will see more conditions that are required of $f$ for (1.7) to have a weak solution. The most important of these will be the behavior of $f$ near zero. Recall that under inverse mean curvature flow, an application of the maximum principle shows that one has an a priori upper bound on $H$ ([8]; 1.4). This will be the case for solutions to (1.1); however, our main concern is not when $H$ is large, but rather when it approaches zero, causing the speed to go to infinity. Our method for dealing with these singularities is entirely inspired by the work of Huisken-Ilmanen [8], where an energy-minimization principle is used to allow the surfaces to jump when this energy minimization is not satisfied.

Let us now record some basic formulae that follow for a solution to (1.1). First of all, for a family of hypersurfaces with outward normal speed $v$ we have the following general formulae:

$$
\begin{aligned}
\frac{\partial}{\partial t} d \mu_{t} & =H v d \mu_{t}, \\
\frac{\partial H}{\partial t} & =\Delta(-v)-|A|^{2} v-\operatorname{Rc}(\nu, \nu) v .
\end{aligned}
$$

Thus for a solution to (1.1) we have immediately:

$$
\frac{\partial}{\partial t} d \mu_{t}=\frac{H}{f(H)} d \mu_{t}
$$

and

$$
\begin{aligned}
\frac{\partial H}{\partial t} & =\Delta\left(-\frac{1}{f(H)}\right)-\frac{|A|^{2}}{f(H)}-\frac{\operatorname{Rc}(\nu, \nu)}{f(H)} \\
& =\frac{f^{\prime}(H) \Delta H}{f(H)^{2}}+\frac{f^{\prime \prime}(H)|\nabla H|^{2}}{f(H)^{2}}-2 \frac{f^{\prime}(H)^{2}|\nabla H|^{2}}{f(H)^{3}}-\frac{|A|^{2}}{f(H)}-\frac{\operatorname{Rc}(\nu, \nu)}{f(H)} .
\end{aligned}
$$

We now give an outline of the rest of the paper. As we mentioned before and is clear when reading the paper, we broadly adapt the work of Huisken-Ilmanen [8]. In Section 2, we reformulate solutions to (1.1) using an energy-minimization principle directly generalizing the one found in [8]. We also prove various general analytic properties of solutions to (1.1) and in 
the process identify various conditions on $f$ which will be helpful in proving existence and uniqueness. In Section 3, we define a very general condition on $f$ which guarantees existence and uniqueness of solutions to (1.1) with precompact initial condition. In Section 4, we restrict to the case where $f$ is given by $F^{\prime}$ where $F^{\prime}$ is the solution to (1.3). We closely follow [8] and generalize the monotonicity of the Hawking mass for weak solutions to inverse mean curvature flow to give the monotonicity of (1.2) under the generalized flow. Finally in Section 5, we prove asymptotic convergence of the surface at infinity to a round sphere, which completes the proof of Theorem 1.1.

\section{Variational formulation}

\subsection{Weak solutions}

Let $f: \mathbb{R} \rightarrow \mathbb{R}$ be strictly monotonically increasing. Generalizing the work of Huisken-Ilmanen [8] to our setting, consider the following functional:

$$
J_{u}(v)=J_{u}^{K}(v)=\int_{K}|\nabla v|+v f^{-1}(|\nabla u|) d x .
$$

Note that as we have assumed that $f(x)$ is strictly monotonically increasing for positive $x, f^{-1}$ is well defined. The Euler-Lagrange equation of this functional is

$$
\operatorname{div}_{M} \frac{\nabla v}{|\nabla u|}=f^{-1}|\nabla u|
$$

or equivalently

$$
f\left(\operatorname{div}_{M} \frac{\nabla v}{|\nabla u|}\right)=|\nabla u| .
$$

Definition 2.1. Let $u$ be a locally Lipschitz function on the open set $\Omega$. Then $u$ is a weak solution (subsolution, supersolution respectively) of (1.7) if

$$
J_{u}^{K}(u) \leq J_{u}^{K}(v)
$$

for every locally Lipschitz function $v(v \leq u, v \geq u$, respectively) such that $\{v \neq u\} \subset \subset \Omega$, where the integration is performed over any compact set $K$ containing $\{u \neq v\}$. 


\subsection{Isoperimetric reformulation}

In this subsection we again adapt [8] and reformulate the equation in terms of a modified isoperimetric problem. Let $F$ be a set of locally finite perimeter, and let $\partial^{*} F$ denote its boundary. Then given $u$ locally Lipschitz and $K \subset$ $M$ define:

$$
J_{u}(F)=J_{u}^{K}(F)=\left|\partial^{*} F \cap K\right|-\int_{F \cap K} f^{-1}(|\nabla u|) .
$$

This functional can be interpreted as area minimization plus bulk energy term, which will be important in the sequel.

Definition 2.2. We say that $E$ minimizes $J_{u}$ in a set $A$ (on the outside, inside respectively) if

$$
J_{u}^{K}(E) \leq J_{u}^{K}(F)
$$

for every $F$ such that $F \Delta E \subset \subset A$ (with $F \supseteq E, F \subseteq E$ respectively), and any compact set containing $F \Delta E$.

Lemma 2.3. Let $u$ be a locally Lipschitz function in the open set $\Omega$. Then $u$ is a weak solution of (1.7) in $\Omega$ if and only if for each $t, E_{t}=\{u<t\}$ minimizes $J_{u}$ in $\Omega$.

Proof. Let $v$ be a locally Lipschitz function such that $\{v \neq u\} \subset \subset \Omega$ and $K$ a compact set containing $\{v \neq u\}$. Set $E_{t}=\{u<t\}, F_{t}=\{v<t\}$. Since $v=u$ outside of $K$, we have $F_{t} \Delta E_{t} \subseteq K$ for every $t$. So, pick $a<b$ such that $a<u, v<b$ on $k$, and then by the co-area formula we have:

$$
\begin{aligned}
J_{u}^{K}(v) & =\int_{K}|\nabla v|+v f^{-1}|\nabla u| \\
& =\int_{a}^{b} \int_{F_{t}} d A d t-\int_{K} \int_{a}^{b} \chi_{v<t} f^{-1}(|\nabla u|) d t d V+b \int_{K} f^{-1}(|\nabla u|) d V \\
& =\int_{a}^{b} J_{u}^{K}\left(F_{t}\right)+b \int_{K} f^{-1}(|\nabla u|) .
\end{aligned}
$$

Thus if each $E_{t}$ minimizes $J_{u}$ in $\Omega$, then it is clear that $u$ will minimize $J_{u}$ in $\Omega$. This proves one direction. As for the other direction parts 2 and 3 of Lemma 1.1 in [8] give a proof that works for minimizers of a functional which is area minimization plus bulk energy term. 
Lemma 2.4 (Smooth flow lemma). Let $\left\{N_{t}\right\}_{a \leq t<b}$ be a smooth family of surfaces of positive mean curvature solving (1.1) classically. Let $u$ be a level set function for $N_{t}$, and let $E_{t}:=\{u<t\}$. Then for $a \leq t<b, E_{t}$ minimizes $J_{u}$ in $E_{b}-E_{a}$.

Proof. The exterior normal vector $\nu_{u}:=\frac{\nabla u}{|\nabla u|}$ is a smooth unit vector field on $E_{b}-E_{a}$ with $\operatorname{div} \nu_{u}=f^{-1}\left(H_{n_{t}}\right)>0$. Fix $F$ a set of finite perimeter. Then by applying the divergence theorem with calibration $\nu_{u}$ on the set $F-E_{t}$ we see:

$$
\begin{aligned}
\left|\partial E_{t}\right|-\int_{E_{t}} f^{-1}(|\nabla u|) & =\int_{\partial E_{t}} \nu_{\partial E_{t}} \cdot \nu_{u}-\int_{E_{t}} f^{-1}(|\nabla u|) \\
& =\int_{\partial^{*} F} \nu_{\partial^{*} F} \cdot \nu_{u}-\int_{F} f^{-1}(|\nabla u|) \\
& \leq\left|\partial^{*} F\right|-\int_{F} f^{-1}(|\nabla u|) .
\end{aligned}
$$

\subsection{Initial value problem}

We will typically want to start our flow from a given surface in $M$. Here we define the weak flows starting at a particular initial condition.

Definition 2.5. We say that $u$ is a weak solution of (1.7) with initial condition $E_{0}$ if

$$
u \in C_{\operatorname{loc}}^{0,1}(M), E_{0}=\{u<0\} \quad \text { and } u \text { satisfies (2.4) in } M-E_{0} .
$$

Definition 2.6. Let $E_{t}$ be a nested family of open sets in $M$, closed under ascending union. Define $u$ by $E_{t}=\{u<t\} .\left(E_{t}\right)_{t>0}$ is a weak solution of (2.6) with initial condition $E_{0}$ if

(2.8) $u \in C_{\mathrm{loc}}^{0,1}(M)$ and $E_{t}$ minimizes $J_{u}$ in $M-E_{0}$ for each $t>0$.

The issue of the equation being satisfied at $t=0$ is a delicate one. Like the weak flow in [8], $E_{0}$ must be a minimizing hull for this to hold.

\subsection{Regularity}

Let $E$ contain an open set $U$ and minimize the functional

$$
\left|\partial^{*} F\right|+\int_{F} \phi
$$


with respect to $F$ such that $F \supset U, F \Delta E \subset \subset \Omega$. This is the situation for our weak solutions, thus we may apply the following regularity theorem ([8]; Theorem 1.3).

Theorem 2.7 (Regularity theorem). Let $n<8$.

1. If $\partial U$ is $C^{1}$ then $\partial E$ is a $C^{1}$ submanifold of $\Omega$.

2. If $\partial U$ is $C^{1, \alpha}, 0<\alpha \leq 1 / 2$, then $\partial E$ is a $C^{1, \alpha}$ submanifold of $\Omega$. The $C^{1, \alpha}$ estimates depend only on the distance to $\partial \Omega$, ess $\sup |f|, C^{1, \alpha}$ bounds for $\partial U$ and $C^{1}$ bounds (including positive lower bounds) for the metric $g$.

3. If $\partial U$ is $C^{2}$ and $f=0$, then $\partial E$ is $C^{1,1}$, and $C^{\infty}$ where it does not contact the obstacle $U$.

Now let $u$ be a solution of (2.4) with initial condition $E_{0}$. Set

$$
E_{t}:=\{u<t\}, \quad E_{t}^{+}:=\operatorname{int}\{u \leq t\}, \quad N_{t}:=\partial E_{t}, \quad N_{t}^{+}:=\partial E_{t}^{+} .
$$

The regularity theorem shows that $N_{t}$ and $N_{t}^{+}$have locally uniform $C^{1, \alpha}$ estimates depending only on local Lipschitz bounds for $u$. Specifically, for all $t>0$,

$$
N_{s} \rightarrow N_{t} \text { as } s \nearrow t, \quad N_{s} \rightarrow N_{t}^{+} \text {as } s \searrow t
$$

locally in $C^{1, \beta}, 0 \leq \beta<\alpha$. If $\partial E_{0}$ is $C^{1, \alpha}$, this holds as $s \downarrow 0$ also.

\subsection{Minimizing hulls}

Recall that $E$ is a minimizing hull if $E$ minimizes area on the outside, that is, if

$$
\left|\partial^{*} E \cap K\right| \leq\left|\partial^{*} F \cap K\right|
$$

for any $F$ containing $E$, and any $K$ containing $F \backslash E$. $E$ is a strictly minimizing hull if the equality holds only when $F=E$ a.e. Finally recall that for a given measurable set $E, E^{\prime}$ is the strictly minimizing hull of $E$, defined as the intersection of all strictly minimizing hulls that contain $E$. We will need the following basic fact about minimizing hulls: if $\partial E$ is $C^{2}$, part 3 of 
Theorem 2.7, applies to $E^{\prime}$ to show that the weak mean curvature satisfies

$$
\begin{aligned}
& H_{\partial E^{\prime}}=0 \quad \text { on } \partial E^{\prime} \backslash \partial E \\
& H_{\partial E^{\prime}}=H_{\partial E} \geq 0 \quad \mathcal{H}^{n-1}-\text { a.e. } \quad \text { on } \partial E^{\prime} \cap \partial E .
\end{aligned}
$$

See $[8]$ for more background on minimizing hulls. The following proposition shows that the qualitative behavior of our generalized inverse mean curvature flow is very similar to the usual inverse mean curvature flow when $f(0)=0$.

Proposition 2.8. Suppose that u satisfies (2.7) and that $M$ has no compact components, and suppose $f(0)=0$. Then:

1. for $t>0, E_{t}$ is a minimizing hull in $M$;

2. for $t \geq 0, E_{t}^{+}$is a strictly minimizing hull in $M$;

3. for $t \geq 0, E_{t}^{\prime}=E_{t}^{+}$, provided $E_{t}^{+}$is precompact;

4. for $t>0,\left|\partial E_{t}\right|=\left|\partial E_{t}^{+}\right|$provided that $E_{t}^{+}$is precompact. This extends to $t=0$ precisely if $E_{0}$ is a minimizing hull.

Proof. 1. First note that because we assume $f$ is monotonically increasing, the assumption $f(0)=0$ implies that $f(x)>0$ for $x>0$. Using Equation (2.8) we have:

$$
\left|\partial E_{t} \cap K\right|+\int_{F-E_{t}} f^{-1}(|\nabla u|) \leq\left|\partial^{*} F \cap K\right|
$$

for $t>0$, and any $F$ containing $E_{t}$. Note that we use the positivity assumption on $f$ to conclude that the second term shown is positive. This then exactly says that $E_{t}$ is a minimizing hull for $t>0$.

2. As before we have the following equation:

$$
\left|\partial E_{t}^{+} \cap K\right|+\int_{F-E_{t}^{+}} f^{-1}(|\nabla u|) \leq\left|\partial^{*} F \cap K\right|
$$

for $t \geq 0$ and appropriate $F$ and $K$. This shows as before that $E_{t}^{+}$is a minimizing hull. To show that it is in fact strictly minimizing, pick $F \supset$ $E_{t}^{+}$with

$$
\left|\partial^{*} F \cap K\right|=\left|\partial E_{t}^{+} \cap K\right| .
$$

Then by (2.12) and our assumptions on $f$ we have that $\nabla u=0$ a.e. on $F-E_{t}^{+}$; thus, it is clear that $F$ is a minimizing hull. By general properties 
of minimizing hulls we may assume that $F$ is open. Then we have that $u$ is constant on each connected component of $F-\bar{E}_{t}^{+}$. Since $F$ is a minimizing hull, any component with closure disjoint from $\bar{E}_{t}^{+}$must have no perimeter, and thus be both open and closed. Since $M$ has no compact components this is impossible. Thus $u=t$ on $F-E_{t}^{+}$so that $F \subset E_{t}^{+}$and hence $E_{t}^{+}$is strictly minimizing.

3. By 2. we have $E_{t}^{\prime} \subseteq E_{t}^{+}$. If they are not equal and $E_{t}^{+}$is precompact then $\left|\partial E_{t}^{\prime}\right|<\left|\partial E_{t}^{+}\right|$which contradicts (2.12).

4. If $E_{t}^{+}$is precompact then $E_{t}$ is as well so we may use them as test sets in (2.11) and (2.12) to conclude $\left|\partial E_{t}\right| \leq\left|\partial E_{t}^{+}\right| \leq\left|\partial E_{t}\right|$ which proves the condition for $t>0$. These inequalities hold for $t=0$ only if $E_{0}$ itself happens to be a minimizing hull.

Lemma 2.9 (Growth lemma). Let $\left(E_{t}\right)$ solve (2.6) with initial condition $E_{0}$. Then for $t>0$, as long as $E_{t}$ remains precompact, we have:

$$
\frac{\partial}{\partial t}\left|\partial E_{t}\right|=\int_{E_{t}} \frac{f^{-1}(|\nabla u|)}{|\nabla u|} .
$$

Proof. By the minimizing property, we know that $J_{u}\left(E_{t}\right)$ is constant for all time. So, using the co-area formula we see:

$$
\begin{aligned}
0=\frac{\partial}{\partial t} J\left(E_{t}\right) & =\frac{\partial}{\partial t}\left(\left|\partial E_{t}\right|-\int_{E_{t}} f^{-1}(|\nabla u|)\right) \\
& =\frac{\partial}{\partial t}\left(\left|\partial E_{t}\right|-\int_{0}^{t} \int_{\partial E_{\tau}} \frac{f^{-1}(|\nabla u|)}{|\nabla u|} d A d \tau\right)
\end{aligned}
$$

and the result follows.

We will also need the following corollary specific to the choice of $F$ given by $(1.3)$.

Corollary 2.10. Let $\left(E_{t}\right)$ solve $(2.6)$ with initial condition $E_{0}$, a minimizing hull where $f=F^{\prime}$ with $F$ given by (1.3). Then $\left|\partial E_{t}\right| \geq e^{t}\left|\partial E_{0}\right|$.

Proof. An argument similar to the previous lemma applies to $\left|\partial E_{t}^{+}\right|$for $t \geq$ 0 . We note that for $F$ given by (1.3) we have $\frac{f^{-1}(|\nabla u|)}{|\nabla u|} \geq 1$ so that $\frac{\partial}{\partial t}\left|\partial E_{t}^{+}\right| \geq$ $\left|\partial E_{t}^{+}\right|$. We note that $f(0)=0$ so that if $E_{0}$ is minimizing Proposition 2.8 (part 4) gives the result. 


\subsection{Compactness}

Theorem 2.11. Let $\left\{u_{i}\right\}$ be a sequence of solutions to (2.4) on open sets $\Omega_{i}$ in $M$ such that

$$
u_{i} \rightarrow u, \quad \Omega_{i} \rightarrow \Omega
$$

locally uniformly, and for each $K \subset \subset \Omega$,

$$
\sup _{K}\left|\nabla u_{i}\right| \leq C(K)
$$

for sufficiently large $i$. Also assume that

$$
\lim _{x \rightarrow 0^{+}} f^{\prime}(x)>0 .
$$

Then $u$ is a solution of (2.4) on $\Omega$.

Proof. We prove this theorem by a form of induction. Let $v$ be a locally Lipschitz function such that $\{v \neq u\} \subset \subset \Omega$. We want to show that $J_{u}(u) \leq$ $J_{u}(v)$. Since the set $\{v \neq u\}$ is compact, and we are assuming a uniform bound on the derivatives of the $u_{i}$ on compact sets, there exists $s$ bounding all of these derivatives on this set. By (2.13), we see that there exists a constant $C_{f}$ such that $f^{-1}(x) \leq C_{f} x$ on $[0, C(K)]$. First assume that $v<u+\frac{1}{C_{f}}$. Let $\phi \in C_{c}^{1}(\Omega)$ be a cutoff function such that $\phi=1$ on $\{v \neq u\}$. Then define

$$
v_{i}=\phi v+(1-\phi) u_{i} .
$$

Clearly $v_{i}$ is a valid comparison function for $u_{i}$ in the sense of definition (2.1). Let $U$ be a small neighborhood containing the support of $\phi$. Then by Equation (2.4), we have for sufficiently large $i$ :

$$
\begin{aligned}
\int_{U}\left|\nabla u_{i}\right|+u_{i} f^{-1}\left(\left|\nabla u_{i}\right|\right) \leq & \int_{U}\left|\nabla v_{i}\right|+v_{i} f^{-1}\left(\left|\nabla u_{i}\right|\right) \\
= & \int_{U}\left|\phi \nabla v+(1-\phi) \nabla u_{i}+\nabla \phi\left(v-u_{i}\right)\right| \\
& +\left(\phi v+(1-\phi) u_{i}\right) f^{-1}\left(\left|\nabla u_{i}\right|\right) \\
\leq & \int \phi|\nabla v|+(1-\phi)\left|\nabla u_{i}\right|+\left|\nabla \phi\left(v-u_{i}\right)\right| \\
& +\left(\phi v+(1-\phi) u_{i}\right) f^{-1}\left(\left|\nabla u_{i}\right|\right) .
\end{aligned}
$$


Thus, rearranging we get:

$$
\int_{U} \phi\left(\left|\nabla u_{i}\right|+\left(u_{i}-v\right) f^{-1}\left(\left|\nabla u_{i}\right|\right)\right) \leq \int_{U} \phi|\nabla v|+\left|\nabla \phi\left(v-u_{i}\right)\right| .
$$

Note that in the limit the last term in this equation goes to zero. This is because eventually either $v=u$ or $\nabla \phi=0$ at every point. But now

$$
\begin{aligned}
v & <u+\frac{1}{C_{f}} \\
& <u+\frac{\left|\nabla u_{i}\right|}{f^{-1}\left(\left|\nabla u_{i}\right|\right)} .
\end{aligned}
$$

Thus for large enough $i$ we have $1+\left(u_{i}-v\right) \frac{f^{-1}\left(\left|\nabla u_{i}\right|\right)}{\left|\nabla u_{i}\right|}$ is positive. Thus, by lower semicontinuity we may pass to the limit to achieve:

$$
\int_{U} \phi\left(|\nabla u|\left(1+(u-v) f^{-1}(|\nabla u|)\right) \leq \int_{U} \phi|\nabla v| .\right.
$$

This completes the first stage of the induction. Now we must prove that if $u$ satisfies (2.4) for all $w \leq u+\epsilon$ then it does for each $v \leq u+2 \epsilon$. This follows exactly as in Theorem 2.1 of [8], using the inequalities $J_{u}(u) \leq J_{u}\left(v_{i}\right)$ where $v_{1}=\min (v, u+k), v_{2}=\max (v-k, u)$.

\subsection{Uniqueness}

In this subsection we give uniqueness properties for solutions to (1.7). Theorem 2.12 follows the proof of uniqueness given in [8], but does not cover all the cases we need. Therefore, we resort to using the approximation scheme we use in Section 3 to cover the remaining case.

Theorem 2.12. Assume that $M$ has no compact components, and assume

$$
0<\lim _{x \rightarrow 0^{+}} f^{\prime}(x)<\infty
$$

1. If $u$ and $v$ solve (2.4) on an open set $\Omega$ in $M$, and $\{v>u\} \subset \subset \Omega$, then $v \leq u$ on $\Omega$.

2. If $\left(E_{t}\right)_{t>0}$ and $\left(F_{t}\right)_{t>0}$ solve (2.8) in a manifold $M$ and the initial conditions satisfy $E_{0} \subset F_{0}$, then $E_{t} \subset F_{t}$ as long as $E_{t}$ is precompact in $M$. 
3. In particular, for a given $E_{0}$ there exists at most one solution $\left(E_{t}\right)_{t>0}$ of (2.8) such that each $E_{t}$ is precompact.

Proof. First we note that assumption (2.14) implies that the derivative of $f^{-1}$ is bounded below, so that on any compact set $I \subset \mathbb{R}_{\geq 0}$ there exists a constant $C_{f}$ such that:

$$
|x-y| \leq C_{f}\left|f^{-1}(x)-f^{-1}(y)\right|
$$

for all $x, y \in I$. This is the property that we will use in the following proof.

(1) First we assume that $u$ is a strict weak supersolution of (2.4), i.e., for any Lipschitz function $w \geq u$ with $\{w \neq u\} \subset \subset \Omega$ we have:

$$
\int|\nabla u|+u f^{-1}(|\nabla u|)+\epsilon \int(w-u) f^{-1}(|\nabla u|) \leq \int|\nabla w|+w f^{-1}(|\nabla u|)
$$

for some $\epsilon>0$. Use this formula with $w=u+(v-u)_{+}$to get:

$\int_{v>u}|\nabla u|+u f^{-1}(|\nabla u|)+\epsilon \int_{v>u}(v-u) f^{-1}(|\nabla u|) \leq \int_{v>u}|\nabla v|+v f^{-1}(|\nabla u|)$.

As we have assumed that $v$ is a solution of (2.4), use the equation $J_{v}(v) \leq$ $J_{v}(\min \{v, u\})$ to get:

$$
\int_{v>u}|\nabla v|+v f^{-1}(|\nabla v|) \leq \int_{v>u}|\nabla u|+u f^{-1}(|\nabla v|) .
$$

Adding the previous two equations together gives:

$$
\int_{v>u}(v-u)\left(f^{-1}(|\nabla v|)-f^{-1}(|\nabla u|)\right)+\epsilon \int_{v>u}(v-u) f^{-1}(|\nabla u|) \leq 0 .
$$

Now we want to bound the term $(v-u) f^{-1}(|\nabla u|)$. Again we will use the minimizing property of $u$. As before, use the equation $J_{u}(u) \leq J_{u}(u+(v-$ $s-u)_{+}$) where $s \geq 0$ and then integrate over $s$ to get:

$$
\begin{aligned}
& \int_{0}^{\infty} \int_{v-s>u}|\nabla u|+u f^{-1}(|\nabla u|) d x d s \\
& \quad \leq \int_{0}^{\infty} \int_{v-s>u}|\nabla v|+(v-s) f^{-1}(|\nabla u|) d x d s .
\end{aligned}
$$


Switching the order of integration gives

$$
\begin{aligned}
& \int_{M}|\nabla u| \int_{-\infty}^{\infty} \chi_{\{s>0\}} \chi_{\{v-u>s\}}\left(1+(u-v+s) \frac{f^{-1}(|\nabla u|)}{|\nabla u|}\right) d s d x \\
& \quad \leq \int_{M}|\nabla v| \int_{-\infty}^{\infty} \chi_{\{s>0\}} \chi_{\{v-u>s\}} d s d x
\end{aligned}
$$

which after integrating yields

$$
\int_{v>u}-\frac{(v-u)^{2}}{2} f^{-1}(|\nabla u|) \leq \int_{v>u}(v-u)(|\nabla v|-|\nabla u|) .
$$

Putting this equation into (2.16) and applying our assumption on $f$ we see that

$$
-\frac{1}{C_{f}} \int_{v>u} \frac{(v-u)^{2}}{2} f^{-1}(|\nabla u|)+\epsilon \int_{v>u}(v-u) f^{-1}(|\nabla u|) \leq 0 .
$$

Assuming $v \leq u+C_{f} \epsilon$; the previous inequality then implies that $|\nabla u|=0$ a.e. on $\{v>u\} ;(2.15)$ then implies that $|\nabla v|=0$ on $\{v>u\}$. We then conclude that since $M$ has no compact component, $v \leq u+C_{f} \epsilon$ implies $v \leq u$. For general $v$ we simply subtract a constant to contradict the one shown. Now if $u$ is a general weak supersolution we make the approximation $u^{\epsilon}=\frac{u}{(1-\epsilon)}$ by strict supersolutions and apply the one shown.

(2) Another property that our flow shares with usual inverse mean curvature flow is that if $u$ is a weak solution, so is $u^{t}=\min (u, t)$ for every $t \in \mathbb{R}$. Using this, let $W:=E_{t}-\bar{F}_{0}$, a precompact open set. Since $E_{0} \subset F_{0}$, we have $v^{t}<u+\delta$ near $\partial W$, thus $\left\{v^{t}>u+\delta\right\}$ is compact in $W$, so condition (1) implies that $v^{t} \leq u+\delta$ on $W$, hence $v^{t} \leq u$ on $W$. Since $u<t$ on $W$, $v \leq u$ on $W$, hence $E_{t} \subset F_{t}$.

(3) is immediate from $(2)$.

So, for the case $f(x)=x^{\alpha}, \alpha<1$, a new proof is needed. We will use the approximation scheme by smooth solutions used in the existence proof in Section 3 to prove uniqueness in this case.

Corollary 2.13. Assume that $M$ has no compact components, and assume

$$
0<\lim _{x \rightarrow 0^{+}} f^{\prime}(x)
$$

Then all of the conclusions of Theorem 2.12 hold. 
Proof. Assumption (2.18) now implies that for every compact set $I \subset \mathbb{R}_{>0}$ there exists $C_{f}$ such that for all $x, y \in I$, we have

$$
|x-y| \leq C_{f}\left|f^{-1}(x)-f^{-1}(y)\right| .
$$

So, for compact regions of smooth solutions $u, v$ we have the existence of $x_{0}$ such that $|\nabla u|,|\nabla v| \geq x_{0}>0$. Thus, we may repeat the earlier proof of (1) to get line (2.17) using $C_{f}$, and hence we prove part (1) for smooth solutions. The same approximation scheme by cylinders used in [8] will be used in our proof of existence. In particular for any solution $u$ over an open set $\Omega$ there exists a sequence of approximate smooth solutions $U^{\epsilon}=u^{\epsilon}-\epsilon z$ where $U^{\epsilon}$ solves (1.7) smoothly on $\Omega \times \mathbb{R}$ and $u^{\epsilon} \rightarrow u$ locally uniformly. So let $v^{\epsilon}$ and $u^{\epsilon}$ be these smooth approximate solutions. It is clear that for $\epsilon$ small we may further choose a small $\delta>0$ such that if we define $u_{\delta}^{\epsilon}:=\frac{u^{\epsilon}}{1-\delta}$ then $\left\{v^{\epsilon}>u_{\delta}^{\epsilon}\right\} \subset \subset \Omega$, and $\delta \rightarrow 0$ as $\epsilon \rightarrow 0$. Thus, we may apply the earlier result on smooth (super)solutions to conclude $v^{\epsilon} \leq u_{\delta}^{\epsilon}$. Letting $\epsilon \rightarrow 0$ gives part (1). The other parts follow formally, as they did not rely on the behavior of $f$ near zero.

\section{Existence}

In this Section, we will prove existence of solutions to the initial value problem (2.7) via elliptic regularization, the method employed in [8]. Recall that $u$ is proper if every $\{s \leq u \leq t\}$ is compact. Set $H_{+}=\max \left(0, H_{\partial E_{0}}\right)$. We will go ahead and collect all of our previous assumptions on $f$, and make a delicate assumption on the growth of $f$ near 0 :

Definition 3.1. A function $f: \mathbb{R}^{+} \rightarrow \mathbb{R}$ satisfies condition $A$ if:

1. $f \in C^{1}$;

2. $f^{\prime}(x)>0$ for all $x>0$;

3. $f(0)=0$;

4. there exists $\epsilon>0, C_{f}>0$ and $0<\alpha \leq 1$ so that for every $x \in[0, \epsilon)$ we have

$$
\begin{aligned}
\frac{1}{C_{f}} x^{\alpha} & \leq f(x) \leq C_{f} x^{\alpha} \\
\frac{1}{C_{f}} x^{\alpha-1} & \leq f^{\prime}(x) \leq C_{f} x^{\alpha-1} ;
\end{aligned}
$$


5. there exists $C_{f}>0$ and $0<\alpha$ so that

$$
\begin{gathered}
\frac{1}{C_{f}}<\lim _{x \rightarrow \infty} \frac{f(x)}{x^{\alpha}}<C_{f} \\
\frac{1}{C_{f}}<\lim _{x \rightarrow \infty} \frac{f^{\prime}(x)}{x^{\alpha-1}}<C_{f} .
\end{gathered}
$$

We have already seen the reasons for most of these different restrictions. Condition (2) is necessary for ellipticity (Proposition 1.3) and condition (3) gave us the nice behavior of minimizing hulls (Proposition 2.8). Note that condition (4) in particular implies that $\lim _{x \rightarrow 0^{+}} f^{\prime}(x)>0$, a condition that came up in the proofs of compactness and uniqueness. The growth conditions (4) and (5) will be used to prove a decay estimate on the mean curvature along the flow.

Theorem 3.2. Let $M$ be a complete, connected Riemannian n-manifold without boundary and suppose $f$ satisfies condition A. Suppose there exists a proper locally Lipschitz weak subsolution of (2.7) with a precompact initial condition. Then for any non-empty precompact smooth open set $E_{0}$ in $M$, there exists a proper locally Lipschitz solution u of (2.7) with initial condition $E_{0}$. Moreover, u satisfies:

$$
\left|\nabla u^{\epsilon, \tau}(x)\right| \leq \sup _{\partial E_{0} \cap B_{r}(x)} H_{+}+\frac{C(n)}{r} .
$$

So, let $v$ be the given subsolution, and define $F_{L}=\{v<L\}$. Without loss of generality we may assume that $E_{0} \subset F_{0}$. The region $F_{L}-\bar{E}_{0}$ is precompact. Now, we define the approximate equation $\left(*_{\epsilon}\right)$ :

$$
\begin{aligned}
E^{\epsilon} u^{\epsilon} & =f\left(\operatorname{div}\left(\frac{\nabla u^{\epsilon}}{\sqrt{\left|\nabla u^{\epsilon}\right|^{2}+\epsilon^{2}}}\right)\right)-\sqrt{\left|\nabla u^{\epsilon}\right|^{2}+\epsilon^{2}}=0 \quad \text { in } \Omega_{L}, \\
u^{\epsilon} & =0 \quad \text { on } \partial E_{0}, \\
u^{\epsilon} & =L-2 \quad \text { on } \partial F_{L} .
\end{aligned}
$$

\subsection{Mean curvature estimate}

In this Section, we prove a key interior estimate on the mean curvature for solutions to (1.7). The proof is closely adapted from the corresponding mean curvature estimate in [8]. 
Definition 3.3. For $x \in M$, let $\sigma(x) \in(0, \infty]$ be the supremum of radii $r$ such that $B_{r}(x) \subset \subset M$,

$$
\mathrm{Rc} \geq-\frac{1}{100 n r^{2}} \quad \text { in } B_{r}(x)
$$

and there exists a $C^{2}$ function $p$ on $B_{r}(x)$ such that

$$
p(x)=0, \quad p \geq d_{x}^{2} \text { in } B_{r}(x)
$$

and

$$
|\nabla p| \leq 3 d_{x} \text { and } \nabla^{2} p \leq 3 g \text { on } B_{r}(x)
$$

where $d_{x}$ denotes the distance to $x$.

Proposition 3.4 (Interior estimate of $H$ ). Let $\left(N_{t}\right)_{0 \leq s \leq t}$ smoothly solve (1.7) in $M$, where $f$ satisfies condition $A$ and where $N_{t}$ may have boundary. Then for each $x \in N_{t}$ and each $r<\sigma(x)$ we have

$$
H(x, t) \leq \max \left(H_{r}, \frac{C\left(n, f, \epsilon_{0}\right)}{r}\right)
$$

where $H_{r}$ is the maximum of $H$ on the parabolic boundary of the intersection of the flow with $B_{r}(x), 0 \leq s \leq t$.

Proof. Fix $x$ and $0<r<\sigma(x)$ where $\sigma(x)$ is as before. Let $\psi=\frac{1}{H}$ and then we have the following evolution equation which follows from (1.9):

$$
\begin{aligned}
\frac{\partial \psi}{\partial t} & \geq \frac{f^{\prime}(H)}{f(H)^{2}} \Delta \psi+\frac{\psi^{2}|A|^{2}}{f(H)}+\frac{\psi^{2} \operatorname{Rc}(\nu, \nu)}{f(H)} \\
& \geq \frac{f^{\prime}(H)}{f(H)^{2}} \Delta \psi+\frac{1}{(n-1) f(H)}-\frac{\psi^{2}}{100 n r^{2} f(H)} \\
& =\frac{f^{\prime}\left(\frac{1}{\psi}\right)}{f\left(\frac{1}{\psi}\right)^{2}} \Delta \psi+\frac{1}{(n-1) f\left(\frac{1}{\psi}\right)}-\frac{\psi^{2}}{100 n r^{2} f\left(\frac{1}{\psi}\right)} .
\end{aligned}
$$

We will attempt to find a subsolution $\phi$ of (3.5) that vanishes on $\partial B_{r}$. Now, assuming that $\phi \leq r$, clearly it suffices that:

$$
\frac{\partial \phi}{\partial t} \leq \frac{f^{\prime}\left(\frac{1}{\phi}\right)}{f\left(\frac{1}{\phi}\right)^{2}} \Delta \phi+\frac{1}{2 n f\left(\frac{1}{\phi}\right)} .
$$


We now state some formulae for the ambient derivatives of $\phi$ :

$$
\frac{\partial \phi}{\partial t}=\frac{1}{f\left(\frac{1}{\psi}\right)} \nu \cdot \nabla \phi, \quad \Delta \phi=\operatorname{tr}_{N_{t}}\left(\nabla^{2} \phi\right)-\frac{1}{\psi} \nu \cdot \nabla \phi
$$

where $\Delta$ is the intrinsic Laplacian on $N_{t}$ and $\nabla^{2}$ refers to the global covariant derivative on $M$. Assuming that $\phi<\psi$ initially we can replace $\psi$ by $\phi$ in the previous equations as far as producing a subsolution is concerned. Then plugging these equations into (3.6) gives us the following sufficient condition for a function $\phi$ to be a subsolution of (3.5):

$$
0 \leq \frac{f^{\prime}\left(\frac{1}{\phi}\right)}{f\left(\frac{1}{\phi}\right)^{2}} \operatorname{tr}_{N_{t}} \nabla^{2} \phi-\left(\frac{1}{f\left(\frac{1}{\phi}\right)}+\frac{f^{\prime}\left(\frac{1}{\phi}\right)}{f\left(\frac{1}{\phi}\right)^{2} \phi}\right) \nu \cdot \nabla \phi+\frac{1}{2 n f\left(\frac{1}{\phi}\right)}
$$

Fix $\epsilon>0, C_{f}>0$ and $\alpha$ from A.4. We will define:

$$
\phi(y):=\frac{A}{r}\left(r^{2}-p(y)\right)_{+}
$$

where $A$ is to be determined. Then $\phi=0$ on $\partial B_{r}$, and $\phi \leq r$ provided $A \leq 1$. We will divide our region into the two cases $\phi \leq \frac{1}{\epsilon}$ and $\phi>\frac{1}{\epsilon}$. First assume $\phi>\frac{1}{\epsilon}$. Then we have

$$
f^{\prime}\left(\frac{1}{\phi}\right) \leq 2 c_{2} \phi^{-\alpha+1}, \quad f\left(\frac{1}{\phi}\right)^{2} \geq\left(2 c_{1} \phi^{-\alpha}\right)^{2}, \quad \frac{f^{\prime}\left(\frac{1}{\phi}\right)}{f\left(\frac{1}{\phi}\right)^{2}} \leq \frac{c_{2} \phi^{\alpha+1}}{4 c_{1}^{2}}
$$

Also, note that $|\nabla \phi|=\frac{A}{r}|\nabla p| \leq 3 A$. Let $C(f)$ denote different constants depending only on the constants $c_{1}$ and $c_{2}$. Then $\frac{1}{f\left(\frac{1}{\phi}\right)}|\nabla \phi| \leq 3 C(\epsilon) A \phi^{\alpha}$ and similarly $\frac{f^{\prime}\left(\frac{1}{\phi}\right)}{f\left(\frac{1}{\phi}\right)^{2} \phi}|\nabla \phi| \leq C(\epsilon) A \phi^{\alpha}$. We also have $\phi^{\alpha+1} \operatorname{tr}_{N_{t}}\left(\nabla^{2} \phi\right) \leq \phi^{\alpha} n A^{2}$ using $\phi \leq r$. Thus we see that $\phi$ is a subsolution if $c(n) C(f) A \leq \frac{1}{2 n}$. Clearly there exists $A \leq 1$ satisfying this inequality.

Now we must deal with the other region $\phi \leq \frac{1}{\epsilon}$. Using conditions A.5 and A.1 it is clear that there exists a new $0<\alpha \leq 1$ such that:

$$
f^{\prime}\left(\frac{1}{\phi}\right) \leq c_{4} \phi^{1-\alpha}, \quad f\left(\frac{1}{\phi}\right)^{2} \geq\left(c_{3} \phi^{-\alpha}\right)^{2}, \quad \frac{f^{\prime}\left(\frac{1}{\phi}\right)}{f\left(\frac{1}{\phi}\right)^{2}} \leq a \phi^{1+\alpha}
$$


where $a$ is determined by $c_{4}, c_{3}$. Thus our condition for $\phi$ to be a subsolution on this region becomes

$$
0 \leq a \phi^{1+\alpha} \operatorname{tr}_{N_{t}}\left(\nabla^{2} \phi\right)-a \phi^{\alpha} \nu \cdot \nabla \phi+\frac{\phi^{\alpha}}{2 c_{4} n} .
$$

Dividing out by $\phi^{\alpha}$ we see that this is equivalent to:

$$
\frac{1}{2 c_{4} n} \geq a \phi\left|\operatorname{tr}_{N_{t}}\left(\nabla^{2} \phi\right)\right|+a|\nabla \phi| .
$$

Using argument similar to the previous paragraph it is clear that this inequality can be achieved by a small choice of $A$ depending only on $f$ and $n$. A straightforward application of the maximum principle as in [8] using this subsolution $\phi$ proves the result.

\subsection{Approximate solutions}

We will now proceed to approximate equation $\left(*_{\epsilon}\right)$ by the following family of equations $\left(*_{\epsilon, \tau}\right)$.

$$
\begin{aligned}
E^{\epsilon} u^{\epsilon, \tau} & =f\left(\operatorname{div}\left(\frac{\nabla u^{\epsilon, \tau}}{\sqrt{\left|\nabla u^{\epsilon, \tau}\right|^{2}+\epsilon^{2}}}\right)\right)-\sqrt{\left|\nabla u^{\epsilon, \tau}\right|^{2}+\epsilon^{2}}=0 \text { in } \Omega_{L}, \\
u^{\epsilon, \tau} & =0 \quad \text { on } \partial E_{0}, \\
u^{\epsilon, \tau} & =\tau \quad \text { on } \partial F_{L} .
\end{aligned}
$$

for $0 \leq \tau \leq L-2$. The idea of course is that we will build our solution to $\left(*_{\epsilon}\right)$ up from the zero solution.

Lemma 3.5 (Estimates on $u^{\epsilon, \tau}$ ). Suppose the subsolution $v$ provided in Theorem 3.2 is smooth, with $\nabla v \neq 0$. Then for every $L>0$ there is an $\epsilon(L)>0$ such that for $0<\epsilon \leq \epsilon(L)$ and $0 \leq \tau \leq L-2$, a smooth solution of $\left(*_{\epsilon, \tau}\right)$ satisfies:

$$
\begin{gathered}
u^{\epsilon, \tau} \geq-\epsilon \text { on } \bar{\Omega}_{L}, u^{\epsilon, \tau} \geq v+\tau-L \text { on } \bar{F}_{L}-F_{0}, \\
\left|\nabla u^{\epsilon, \tau}\right| \leq f\left(H_{+}\right)+\epsilon \text { on } \partial E_{0},\left|\nabla u^{\epsilon, \tau}\right| \leq C(L), \\
\left|\nabla u^{\epsilon, \tau}(x)\right| \leq \max _{\partial \Omega_{L} \cap B_{r}(x)} f^{-1}\left(\sqrt{|\nabla u|^{2}+\epsilon^{2}}\right)+\frac{C(n)}{r^{\alpha(0)}}, \\
\left|u^{\epsilon, \tau}\right|_{C^{2, \alpha}\left(\Omega_{L}\right)} \leq C(\epsilon, L) .
\end{gathered}
$$


Proof. (i) The first step is to define a subsolution that bridges the gap from $E_{0}$ to where $v$ starts. Define $G_{0}=E_{0}$ and $G_{s}:=\left\{x \mid d\left(x, E_{0}\right)<s\right\}$. Pick $s_{L}$ such that $G_{s_{L}} \supset F_{L}$. This is possible because $M$ is connected, $E_{0}$ is nonempty and $E_{0}$ and the initial condition for $V$ are precompact. Let $\Sigma$ be the cut locus of $E_{0}$ in $M$. Then on $M-E_{0}-\Sigma$ the distance function is smooth and each point is connected to $E_{0}$ by a unique length-minimizing geodesic $\gamma$. In a neighborhood of such a geodesic, the level sets $\partial G_{s}$ foliate. Differentiating along such a geodesic gives:

$$
\frac{\partial H}{\partial s}=-|A|^{2}-\operatorname{Rc}(\nu, \nu) \leq C_{1}(L) \quad \text { on } \quad \partial G_{s}-\Sigma, 0 \leq s \leq s_{L} .
$$

Thus

$$
H_{\partial G_{s}} \leq \max _{\partial E_{0}} H_{+}+C_{1} s \leq C_{2}(L) \quad \text { on } \quad \partial G_{s}-\Sigma, 0 \leq s \leq s_{L} .
$$

We will do some computations now for a prospective subsolution

$$
w_{1}(x):=\phi(s)=\phi(d(x, G)) x \in \bar{G}_{s_{L}}-E_{0} .
$$

We want to find a $w$ such that $E^{\epsilon} w \geq 0$. This is equivalent to the folowing quantity being non-negative:

$$
\begin{aligned}
P(\phi) & =\sqrt{\left(\phi^{\prime}\right)^{2}+\epsilon^{2}}\left(\operatorname{div}\left(\frac{\nabla w_{1}}{\sqrt{\left|\nabla w_{1}\right|^{2}+\epsilon^{2}}}\right)\right)-\sqrt{\left(\phi^{\prime}\right)^{2}+\epsilon^{2}} f^{-1}\left(\sqrt{\left(\phi^{\prime}\right)^{2}+\epsilon^{2}}\right) \\
& =\left(g^{i j}-\frac{\left(\phi^{\prime}\right)^{2} \nu^{i} \nu^{j}}{\left(\phi^{\prime}\right)^{2}+\epsilon^{2}}\right) \nabla_{i j}^{2} w_{1}-\sqrt{\left(\phi^{\prime}\right)^{2}+\epsilon^{2}} f^{-1}\left(\sqrt{\left(\phi^{\prime}\right)^{2}+\epsilon^{2}}\right) \\
& \geq C_{2} \phi^{\prime}+\frac{\epsilon^{2} \phi^{\prime \prime}}{\left(\phi^{\prime}\right)^{2}+\epsilon^{2}}-\sqrt{\left(\phi^{\prime}\right)^{2}+\epsilon^{2}} f^{-1}\left(\sqrt{\left(\phi^{\prime}\right)^{2}+\epsilon^{2}}\right) .
\end{aligned}
$$

It is clear by condition A.4 that there exists a constant $C_{f}$ such that on $[0,1], f^{-1}(x) \leq C_{f} x$. Consider the function

$$
\phi(s):=\frac{\epsilon}{A}\left(-1+e^{-A s}\right) .
$$

Note in particular that for $\epsilon \leq \epsilon(A, L):=e^{-A_{s_{L}}}$ we have $\epsilon^{2} \leq\left|\phi^{\prime}\right| \leq \epsilon$. Thus $\phi^{\prime}$ is small and we can use our constant $C_{f}$ above to get that $P(\phi) \geq 0$ 
provided

$$
\left(C_{f}\left[\left(\phi^{\prime}\right)^{2}+\epsilon^{2}\right]-C_{2} \phi^{\prime}\right)\left(\left(\phi^{\prime}\right)^{2}+\epsilon^{2}\right) \leq \epsilon^{2} \phi^{\prime \prime} .
$$

Now if we impose $A=A(L):=4+2 C_{2}$ and that $\epsilon \leq \sqrt{\frac{1}{C_{f}}}$ then

$$
\begin{aligned}
\left(C_{f}\left[\left(\phi^{\prime}\right)^{2}+\epsilon^{2}\right]-C_{2} \phi^{\prime}\right)\left(\left(\phi^{\prime}\right)^{2}+\epsilon^{2}\right) & \leq 2 \epsilon^{2}\left(2 C_{f} \epsilon^{2}+C_{2}\left|\phi^{\prime}\right|\right) \\
& \leq 2 \epsilon^{2}\left(2+C_{2}\left|\phi^{\prime}\right|\right) \\
& \leq \epsilon^{2} \phi^{\prime \prime} .
\end{aligned}
$$

Thus for these choices of $\epsilon$ and $A$ it is clear that $\phi$ is a viscosity subsolution of $E^{\epsilon}$ on all of $G_{s_{L}}-\bar{E}_{0}$. Thus as $u \geq w_{1}$ on the boundary we can apply the maximum principle for viscosity solutions to obtain:

$$
\begin{aligned}
u \geq w_{1} \geq-\epsilon \quad \text { in } \quad \bar{\Omega}_{L}, \\
\frac{\partial u}{\partial \nu} \geq-\epsilon \quad \text { on } \quad \partial E_{0} .
\end{aligned}
$$

(ii) Now we consider the function:

$$
w_{2}:=\frac{L-1}{L} v+\tau-(L-1) .
$$

As we have just rescaled and translated our subsolution $v$, it is clear that $E^{0} w_{2}>0$ on $\bar{F}_{L}-F_{0}$. This domain is compact (since $\nabla v \neq 0$ ) so for all sufficiently small $\epsilon$ we have $E^{\epsilon} w_{2}>0$. Note that since $0 \leq \tau \leq L-2$ we have:

$$
\begin{gathered}
u \geq-\epsilon \geq-1 \geq w_{2} \quad \text { on } \quad \partial F_{0}, \\
u=\tau=w_{2} \quad \text { on } \quad \partial F_{L} .
\end{gathered}
$$

Thus, by the maximum principle

$$
\begin{aligned}
u \geq w_{2} & \geq v+\tau-L \quad \text { on } \quad \bar{F}_{L}-F_{0}, \\
\frac{\partial u}{\partial \nu} & \geq-C(L) \quad \text { on } \quad \partial F_{L} .
\end{aligned}
$$

Clearly we also have that a constant is a supersolution of $\left(*_{\epsilon, \tau}\right)$ so that again by the maximum principle:

$$
\begin{array}{cc}
u \leq \tau & \text { in } \quad \bar{\Omega}_{L}, \\
\frac{\partial u}{\partial \nu} \leq 0 & \text { on } \quad \partial F_{L} .
\end{array}
$$


(iii) Now we want to construct a supersolution along $\partial E_{0}$. Choose a function $w_{3}$ such that:

$$
f\left(H_{+}\right)<\frac{\partial w_{3}}{\partial \nu} \leq f\left(H_{+}\right)+\epsilon .
$$

Clearly then $E w_{3}<0$ on $\partial E_{0}$ and thus for small enough $\delta>0, E w_{3}<0$ on $U:=\left\{0 \leq w_{3} \leq \delta\right\}$. We can now reparameterize $w_{3}$ as follows:

$$
w_{4}:=\frac{w_{3}}{1-w_{3} / \delta} .
$$

We have $\nabla w_{4}=\frac{\nabla w_{3}}{\left(1-w_{3} / \delta\right)^{2}}$ thus $E w_{4}<0$, and $w_{4} \rightarrow \infty$ on $\partial U-\partial E_{0}$. It is clear that for sufficiently small $\epsilon$ (and hence small $\delta$ ), we will have $E^{\epsilon} w_{4}<0$ on $V:=\left\{0 \leq w_{4} \leq L\right\}$. Now by (3.15) we have $u \leq \tau \leq L-2$ so that $u \leq w_{4}$ on $\partial V$. Thus, by the maximum principle $u \leq w_{4}$ on $V$ so that:

$$
\frac{\partial u}{\partial \nu} \leq \frac{\partial w_{4}}{\partial \nu}=\frac{\partial w_{3}}{\partial \nu} \leq f\left(H_{+}\right)+\epsilon \quad \text { on } \quad \partial E_{0} .
$$

It is clear now that putting together Equations (3.11) through (3.17) we have proved Equations (3.7) and (3.8).

(iv) Now let $N_{t}^{\epsilon, \tau}$ denote the level set $\{U=t\}$ of the function $U(x, z):=$ $u^{\epsilon, \tau}(x)-\epsilon z,-\infty<t<\infty$. Equation $\left(*_{\epsilon, \tau}\right)$ says

$$
H_{N_{t}^{\epsilon, \tau}}=f^{-1}\left(\sqrt{|\nabla u|^{2}+\epsilon^{2}}\right)
$$

So in particular we have a smooth solution to $(1.7)$ on $\Omega_{L} \times \mathbb{R}$. Let $\widetilde{B}:=$ $B_{r}^{n+1}(x, z)$ be an $(n+1)$-dimensional ball in $M \times \mathbb{R}$. Since the parabolic boundary of $N_{t}^{\epsilon, \tau}$ just translates of $\Omega_{L}$ and $|\nabla u|$ is independent of $z$, we apply (3.4) to get

$$
\begin{aligned}
f^{-1}\left(\sqrt{|\nabla u|^{2}+\epsilon^{2}}\right) & \leq \sup _{t} \max _{\partial N_{t}^{\epsilon, \tau} \cap \widetilde{B}} f^{-1}\left(\sqrt{|\nabla u|^{2}+\epsilon^{2}}\right)+\frac{C(n)}{r} \\
& \leq \max _{\partial \Omega_{L} \cap B_{r}(x)} f^{-1}\left(\sqrt{|\nabla u|^{2}+\epsilon^{2}}\right)+\frac{C(n)}{r} .
\end{aligned}
$$

This is (3.9). Equations (3.7) and (3.9) allow us to apply the Schauder estimates [7] to conclude (3.10).

Lemma 3.6. Under the hypotheses of Lemma 3.5, a smooth solution of $\left(*_{\epsilon}\right)$ exists. 
Proof. (i) We will use the continuity method applied to $\left(*_{\epsilon, \tau}\right)$ for $0 \leq \tau \leq$ $L-2$. Let us set $u=\epsilon w$ and then $\left(*_{\epsilon, \tau}\right)$ becomes:

$$
F^{\epsilon}(w):=\operatorname{div}\left(\frac{\nabla w}{\sqrt{|\nabla w|^{2}+1}}\right)-f^{-1}\left(\epsilon \sqrt{|\nabla w|^{2}+1}\right)=0
$$

with $w=0$ on $\partial \Omega_{L}$. Now

$$
F: C_{0}^{2, \alpha}\left(\bar{\Omega}_{L}\right) \times \mathbb{R} \rightarrow C^{\alpha}\left(\bar{\Omega}_{L}\right)
$$

defined by $F(w, \epsilon):=F^{\epsilon}(w)$ is $C^{1}$ (recall $f \in C^{1}$ ), and has the solution $F(0,0)=F^{0}(0)=0$. Now the linearization of $F^{0}$ at $w=0$ is given by

$$
\mathcal{D} F_{\mid 0}^{0}=\Delta: C_{0}^{2, \alpha}\left(\bar{\Omega}_{L}\right) \rightarrow C^{\alpha}\left(\bar{\Omega}_{L}\right) .
$$

Since this map is an isomorphism there is a solution of $F^{\epsilon}(w)=0$ for small $\epsilon>0$, hence a solution of $\left(*_{\epsilon, \tau}\right)$ with $\tau=0$. Note that this calculation again used condition A.4, making $\left(f^{-1}\right)^{\prime}(0)<\infty$. (ii) Now we fix $\epsilon$ and vary $\tau$. Let $I$ denote the set of $\tau$ such that $\left(*_{\epsilon, \tau}\right)$ has a solution. $I$ contains 0 by step (i). Also, using the estimates in Lemma 3.5, and the Arzela-Ascoli Theorem, $I \cap[0, L-2]$ is closed. Now we must show that $I$ is open. Let $\pi$ denote the boundary map $u \rightarrow u_{\mid \partial \Omega_{L}}$. Now define

$$
G^{\tau}(u):=\left(E^{\epsilon}(u), \pi(u)-\tau \chi_{\partial F_{L}}\right) .
$$

Then $\left(*_{\epsilon, \tau}\right)$ is equivalent to $G^{\tau}(u)=(0,0)$. Again, the map

$$
F: C^{2, \alpha}\left(\bar{\Omega}_{L}\right) \times \mathbb{R} \rightarrow C^{\alpha}\left(\bar{\Omega}_{L}\right) \times C^{2, \alpha}\left(\partial \Omega_{L}\right)
$$

defined by $F(u, \tau):=G^{\tau}(u)$ is $C^{1}$. The linearization of $G^{\tau}$ at a solution $u$ is given by:

$$
\mathcal{D} G_{\mid u}^{\tau}=\left(\begin{array}{c}
\mathcal{D} E_{\mid u}^{\epsilon} \\
\pi
\end{array}\right): C^{2, \alpha}\left(\bar{\Omega}_{L}\right) \rightarrow C^{\alpha}\left(\bar{\Omega}_{L}\right) \times C^{2, \alpha}\left(\partial \Omega_{L}\right) .
$$

Now

$$
E^{\epsilon}(u)=\left(\nabla_{i} A^{i}(\nabla u)\right)+f^{-1}(B(\nabla u))
$$

where $A$ and $B$ are independent of $u$, so that:

$$
\mathcal{D} E_{\mid u}^{\epsilon}(v)=\left(\nabla_{i} A_{p_{j}}^{i}(\nabla u) \nabla_{j} v\right)+\left(f^{-1}\right)^{\prime}(B(\nabla u)) B_{p_{j}}(\nabla u) \nabla_{j} v .
$$


This equation is linear elliptic; hence, by the existence and uniqueness theory $\mathcal{D} G_{\mid u}^{\tau}$ is an isomorphism. Thus, by the implicit function theorem $I$ is open and $L-2 \in I$, and so the result follows.

Now we proceed to prove the main existence theorem.

Proof of Theorem 3.2. (i) First of all we will assume that $v$ is smooth with non-vanishing gradient. By Lemma 3.6, we have a smooth solution $u^{\epsilon}=$ $u^{\epsilon, L-2}$ of $\left(*_{\epsilon}\right)$ on $\Omega_{L}$, where $\epsilon \rightarrow 0$ as $L \rightarrow \infty$. Using Lemma 3.5, we have that

$$
\begin{aligned}
\left|\nabla u^{\epsilon}\right| & \leq \max _{\partial \Omega_{L} \cap B_{r}(x)} f^{-1}\left(\sqrt{|\nabla u|^{2}+\epsilon^{2}}\right)+\frac{C(n)}{r^{\alpha(0)}} \\
& \leq \max _{\partial E_{0} \cap B_{r}(x)} H_{+}+2 \epsilon+\frac{C(n)}{r^{\alpha(0)}}
\end{aligned}
$$

on every compact subset of $M-E_{0}$ with $L$ large enough which allows us to ignore the derivative of $u$ on $\partial F_{L}$. Thus we may apply the ArzelaAscoli theorem to conclude the existence of sequences $L_{i} \rightarrow \infty, \epsilon_{i} \rightarrow 0$ and a subsequence $u_{i}$ and a locally Lipschitz function $u$ such that $u_{i} \rightarrow u$ locally uniformly on $M-E_{0}$, and $u$ satisfies Equation (3.3). Again using Lemma 3.5 we have that

$$
u \geq 0 \text { in } M-E_{0}, \quad u \rightarrow \infty \text { as } x \rightarrow \infty .
$$

Now note that exactly as in [8] we have that $u^{\epsilon}$ solves $\left(*_{\epsilon}\right)$ if and only if $U^{\epsilon}=u^{\epsilon}-\epsilon z$ solves (1.7) on $M \times \mathbb{R}$. So define $U_{i}(x, z)=u_{i}(x)-\epsilon_{i} z$ and $U(x, z)=u(x)$. Then clearly $U_{i} \rightarrow U$ locally uniformly on $\left(M-E_{0}\right) \times \mathbb{R}$ with local Lipschitz bounds. The level sets of $U_{i}$ smoothly solve (1.1) so that using Lemma $2.4 U_{i}$ satisfies the variational formulation. Then we may apply our compactness theorem to get that $U$ satisfies $(2.4)$ on $\left(M-\bar{E}_{0}\right) \times \mathbb{R}$. It remains to check that $u$ in fact satisfies (2.4) on $M-\bar{E}_{0}$. Let $v$ be a locally Lipschitz function with $\{v \neq u\} \subset \subset M-\bar{E}_{0}$ and fix a compact subset $K \supset\{v \neq u\}$. Define a cutoff function $\phi(z)$ with $\left|\phi_{z}\right| \leq 1, \phi=1$ on $[0, S]$ and $\phi=0$ on $\mathbb{R}-(-1, S+1)$. Then putting $V(x, z):=\phi(z) v(x)$ into the equation $J_{U}(U) \leq J_{U}(V)$ we get

$$
\begin{aligned}
& \int_{K \times[-1, S+1]}|\nabla u|+u f^{-1}(|\nabla u|) d x d z \\
& \quad \leq \int_{K \times[-1, S+1]} \phi|\nabla v|+v\left|\phi_{z}\right|+\phi v f^{-1}(|\nabla u|) d x d z .
\end{aligned}
$$


and then dividing by $S$ and passing $S \rightarrow \infty$ gives the result. Now extend $E_{0}$ to be negative on all of $E_{0}$ so that $E_{0}=\{u<0\}$. This completes the proof in the case of $v$ smooth with $\nabla v \neq 0$. (ii) To finish the proof, we first note that there is a smooth subsolution on any complete Riemannian cone of the form

$$
g_{L}=C s^{2} g_{\partial U_{L}} \oplus d s^{2}
$$

where $U$ is a precompact open set in $M$ given by $\beta \log s$ for some $\beta>0$ if $\alpha(0)=1$ and by $\beta s^{(\alpha(0)-1) / \alpha(0)}$ if $\alpha(0)<1$. We now sketch the argument of existence in Theorem 3.1 (1.390) of [8] where the metric is modified at infinity so as to have a smooth subsolution. Fix $L>0$ and choose an open set $F_{L} \subset \subset U_{L} \subset \subset M$. Modify the metric on $U_{L}$ so that near $\partial U_{L}$, $g_{L}$ is isometric to a Riemannian cone as before. By step 1 we have solutions with respect to these modified metrics. Letting $L \rightarrow \infty$ and using our compactness and uniqueness theorems gives the result with the appropriate gradient bound.

\section{Geroch-type monotonicity}

In this section, we will examine extending the result of Geroch monotonicity for the generalized Hawking mass functionals given by (1.2) to the weak setting. We choose $F$ as defined by (1.3), let $f=F^{\prime}$, and note that in this case $\alpha(0)=1$. For more details on $F$ and monotonicity under the smooth flow see [4]. Our proof follows the proof of monotonicity of the Hawking mass for weak solutions to IMCF found in [8], but will require a few more ingredients, for instance Proposition 4.2. The proof consists of examining the approximation scheme we used to show existence and showing that we can bound the appropriate geometric quantities to send the monotonicity calculation to the limit as $\epsilon \rightarrow 0$. First of all we have a connectedness lemma and a technical proposition which are independent of this particular choice of $f$.

Lemma 4.1 (Connectedness lemma). 1. A solution u of (2.4) has no strict local maxima or minima.

2. Suppose $M$ is connected and simply connected with no boundary and a single asymptotically flat end, and $\left\{E_{t}\right\}$ is a solution with initial condition $E_{0}$. If $\partial E_{0}$ is connected, then $N_{t}$ remains connected as long as it stays compact. 
Proof. (1) Say $u$ has a strict local maximum. Then there is a connected precompact component $E$ of $\{u>t\}$ for some $t$. Define $v$ by $v=u$ on $\Omega-E$ and $v=t$ on $E$. Then (2.4) yields

$$
\int_{E}|\nabla u|+u f^{-1}(|\nabla u|) \leq \int_{E} t f^{-1}(|\nabla u|)
$$

from which it immediately follows that $u \leq t$ on $E$, a contradiction. In the case of a minima, we first of all know that $|\nabla u| \leq C_{E}$ on $E$. As we have seen before, there then exists a constant $C_{f}$ such that $f^{-1}(|\nabla u|) \leq C_{f}|\nabla u|$. Now we may choose $t$ in this construction so that in fact $u>t-\frac{1}{C_{f}}$ on $E$. The equation shown now again gives a contradiction.

(2) We give a sketch of the proof of Theorem 4.2, part (2) in [8] which applies directly to this situation. Fix $t>0$ and let $N_{t}=\{u=t\}$. One can show that $W=\{u<t\}$ is connected if $\partial E_{0}$ is connected. Also $X=\{u>t\}$ is connected. If $N_{t}$ has two components, then one can construct a loop that starts at $\partial E_{0}$, crosses $N_{t}$ via one component and returns via the other. This loop cannot be contracted, a contradiction to $M$ being simply connected.

Proposition 4.2. Fix $p>1$. Say $F: \mathbb{R} \rightarrow \mathbb{R}$ is a real valued function such that for all $x \in \mathbb{R}$ there exists $1 \leq \alpha \leq p$ such that $F(x)=x^{\alpha}$. Then for any measurable function $g \in L^{p}(\Omega)$ on a finite measure space $\Omega$, we have

$$
\int_{\Omega} F(g) \leq C_{F}\|g\|_{L^{p}(\Omega)}^{p} .
$$

Proof. We may approximate $g$ in $L^{p}$ by monotonically increasing step functions $\left\{g_{n}\right\}$. Using Holder's inequality we see that for step functions

$$
\begin{aligned}
\int_{\Omega} F\left(g_{n}\right) & =\sum_{i=1}^{m} \int_{E_{i}} g_{n}(x)^{\alpha_{i}} \\
& \leq \sum_{i=1}^{m} \operatorname{vol}(\Omega)^{1-\frac{\alpha_{i}}{p}} \int_{E_{i}} g_{n}^{p} \\
& \leq C_{F}\left\|g_{n}\right\|_{L^{p}(\Omega)}^{p} .
\end{aligned}
$$

Now, applying Fatou's Lemma we get that $\int F(g) \leq \liminf _{n \rightarrow \infty} \int F\left(g_{n}\right) \leq$ $C_{F} \lim _{n \rightarrow \infty}\left\|g_{n}\right\|_{L^{p}(\Omega)}^{p}=C_{F}\|g\|_{L^{p}(\Omega)}^{p}$.

We now show the monotonicity under the weak flow. Assume that $N_{0}$ is smooth and $M$ has a smooth subsolution at infinity. Both of these 
assumptions will later be removed by an approximation scheme. Let $N_{t}^{\epsilon}:=$ $\left\{z=u^{\epsilon} / \epsilon-t / \epsilon\right\}$ where $u^{\epsilon}:=u^{\epsilon, L-2}$ is an approximator defined on $\Omega_{L}$ as in the proof of Theorem 3.2. We choose a cutoff function $\phi \in C_{c}^{2}(\mathbb{R})$ such that $\phi \geq 0$, spt $\phi \subset[1,5]$, and $\int \phi(z) d z=1$. Fix $T>0$ and $L \geq T+7, \epsilon \leq 1$, so that $\partial N_{t}^{\epsilon}$ is disjoint from $M \times \operatorname{spt} \phi$ for $0 \leq t \leq T$. Then the boundary term disappears in the calculation that follows.

$$
\begin{aligned}
\frac{\partial}{\partial t} \int_{N_{t}^{\epsilon}} \phi(z) F(H) \\
=\int_{N_{t}^{\epsilon}} \phi f(H) \frac{\partial H}{\partial t}+2 F(H) \nabla \phi \cdot \frac{\nu}{f(H)}+2 \phi \frac{H F(H)}{f(H)} \\
=\int_{N_{t}^{\epsilon}} \phi f(H)\left(-\Delta\left(\frac{2}{f(H)}\right)-2 \frac{|A|^{2}}{f(H)}-2 \frac{\operatorname{Rc}(\nu, \nu)}{f(H)}\right) \\
\quad+2 \frac{F(H)}{f(H)} \nabla \phi \cdot \nu+2 \phi \frac{H F(H)}{f(H)} \\
=\int_{N_{t}^{\epsilon}} \phi\left(-2 \frac{|D f(H)|^{2}}{f(H)^{2}}-2|A|^{2}-2 \operatorname{Rc}(\nu, \nu)+2 \frac{H F(H)}{f(H)}\right) \\
\quad-2 D \phi \cdot \frac{D f(H)}{f(H)}+2 \frac{F(H)}{f(H)} \nabla \phi \cdot \nu .
\end{aligned}
$$

And we may write this in integrated form as

$$
\begin{aligned}
\int_{N_{r}^{\epsilon}} \phi F(H)= & \int_{N_{s}^{\epsilon}} \phi F(H) \\
& +\int_{r}^{s} \int_{N_{t}^{\epsilon}} 2 \phi\left(\frac{|D f(H)|^{2}}{f(H)^{2}}+|A|^{2}+\operatorname{Rc}(\nu, \nu)-\frac{H F(H)}{f(H)}\right) \\
& +2 D \phi \cdot \frac{D f(H)}{f(H)}-2 \frac{F(H)}{f(H)} \nabla \phi \cdot \nu .
\end{aligned}
$$

Estimates: Let us now estimate each of these different terms. Specifically we will want to fix $T$ and get estimates that depend on $T$ and are independent of $\epsilon$. By the second part of (3.7) we see that the growth of $u^{\epsilon}$ is determined solely by the subsolution $v$, so that there exists a constant $R(T)$ such that

$$
N_{t}^{\epsilon} \cap(M \times \operatorname{spt}(\phi)) \subset K(T):=\left(B_{R(T)}-E_{0}\right) \times[1,5], 0 \leq t \leq T .
$$


Since $E_{t}^{\epsilon}$ is a minimizing hull, using the definition applied to the perturbation $E_{t}^{\epsilon} \cup K(T)$ gives the inequality

$$
\left|N_{t}^{\epsilon} \cap(M \times \operatorname{spt} \phi)\right| \leq|\partial K(T)|=C(T) .
$$

Using (3.3) and (3.4) and the fact that our domain is compact we conclude

$$
|H| \leq C(T) \text { on } N_{t}^{\epsilon} \cap(M \times \operatorname{spt} \phi) .
$$

Given such a bound and the fact that $f$ is monotonically increasing with $\alpha(0)=1$ we have that

$$
C_{f}(T) H \leq f(H) \leq C_{f}^{\prime}(T) H \quad \text { on } \quad 0 \leq t \leq T .
$$

Thus in particular having bounded the area of integration and the integrand by constants depending only on $T$, we get

$$
\int_{N_{t}^{\epsilon}} \phi \frac{H F(H)}{f(H)}+\phi F(H)+\frac{F(H)}{f(H)} \nabla \phi \cdot \nu \leq C(T) .
$$

Now, using the Cauchy-Schwartz inequality and the arithmetic-geometric mean, we see that

$$
\begin{aligned}
2 D \phi \cdot \frac{D f(H)}{f(H)} & \leq \frac{|D \phi|^{2}}{\phi}+\phi \frac{|D f(H)|^{2}}{f(H)^{2}} \\
& \leq C+\phi \frac{|D f(H)|^{2}}{f(H)^{2}}
\end{aligned}
$$

where the last equation follows since $\phi$ is $C^{2}$ with compact support. Using the previous equation and (4.6), (4.7) and (4.8) we see that

$$
\frac{\partial}{\partial t} \int_{N_{t}^{\epsilon}} \phi F(H) \leq \int_{N_{t}^{\epsilon}}-\phi\left(\frac{|D f(H)|^{2}}{f(H)^{2}}+2|A|^{2}\right)+C(T), 0 \leq t \leq T
$$

But now using these inequalities we see that $\int_{N_{t}^{\epsilon}} \phi F(H)$ is uniformly bounded along $0 \leq t \leq T$ so that the time derivative must also be bounded almost everywhere. Thus if we restrict to a $\phi$ such that $\phi=1$ on $[2,4]$ then

$$
\int_{0}^{T} \int_{N_{t}^{\epsilon} \cap(M \times[2,4])} \frac{|D f(H)|^{2}}{f(H)^{2}}+|D f(H)|^{2}+|A|^{2} \leq C(T) .
$$

Now fix any sequence $\epsilon \rightarrow 0$. Note that the functions $\int_{N_{t}^{\epsilon}} \frac{|D f(H)|^{2}}{f(H)^{2}}+\ldots$, defined on $[0, T]$ are now uniformly bounded in $L^{1}$, so that Fatou's lemma 
implies that the liminf of these functions is also in $L^{1}$, and thus bounded almost everywhere. Hence

$$
\liminf _{i \rightarrow \infty} \int_{N_{t}^{\epsilon_{i}} \cap(M \times[2,4])} \frac{|D f(H)|^{2}}{f(H)^{2}}+|D f(H)|^{2}+|A|^{2}<\infty, \quad \text { a.e. } t \geq 0 .
$$

Convergence: Note that we have subsequences $\epsilon_{i} \rightarrow 0, L_{i} \rightarrow \infty, N_{t}^{i}=$ $N_{t}^{\epsilon_{i}}$ such that

$$
N_{t}^{i} \longrightarrow \widetilde{N_{t}}=N_{t} \times \mathbb{R} \text { locally in } C^{1}, \quad \text { a.e. } t \geq 0 .
$$

We will want to pass the integrated equation (4.3) to the limit. First of all, let us record here the following general convergence result. If $N^{i}$ is a sequence of $C^{1}$ hypersurfaces such that $N^{i} \rightarrow N$ locally in $C^{1}$ and

$$
\sup _{i} \operatorname{ess} \sup _{N^{i}}\left|H_{N_{i}}\right|<\infty
$$

then the limit $H_{N}$ exists weakly as a locally $L^{1}$ function, with a weak convergence

$$
\int_{N^{i}} H_{N^{i}} \nu_{N^{i}} \cdot X \longrightarrow \int_{N} H_{N} \nu_{N} \cdot X, X \in C_{c}^{0}(T M)
$$

and lower semicontinuity:

$$
\begin{aligned}
& \underset{N}{\operatorname{ess} \sup }\left|H_{N}\right| \leq \liminf _{i \rightarrow \infty} \operatorname{ess} \sup _{N^{i}}\left|H_{N_{i}}\right| \\
& \int_{N} \phi\left|H_{N}\right|^{2} \leq \liminf _{i \rightarrow \infty} \int_{N^{i}} \phi\left|H_{N^{i}}\right|^{2} .
\end{aligned}
$$

It is clear that composing the almost everywhere $C^{0}$ functions $H_{N^{i}}$ with any $C^{\infty}$ function $w$ will give a weak definition of $w(H)$. Now, let us first examine the $\int_{N_{t}^{i}} \phi F(H)$ term. Using (4.9) we have that the function $\int_{N_{t}^{i}} \phi F(H)-C(T) t$ is monotonically decreasing. Thus we may choose a diagonal subsequence such that

$$
\lim _{i \rightarrow \infty} \int_{N_{t}^{i}} \phi F(H) \text { exists, a.e. } t \geq 0 .
$$

Now pick a $t$ such that (4.11) and (4.12) hold. Given the local $C^{1}$ convergence, it is a general fact that follows from the implicit function theorem 
that the converging surfaces $N_{t}^{i}$ can be written simultaneously as graphs of $C^{1}$ functions $w_{i}$ over a fixed smooth surface $W$. Thus, we will project all quantities down to this surface for comparison. So, consider as part of Equation (4.11) the bound on $|D f(H)|^{2}$. Given that $H$ is bounded uniformly in $i$ on the domain, it is clear that this then gives us an $H_{1}^{2}$ bound on $H$. Thus, applying Rellich's Theorem, Equation (4.13) and Theorem 3.43 of [2], we conclude that there exists a subsequence such that:

$$
\begin{aligned}
& H_{N_{t}^{i}} \longrightarrow H_{N_{t}} \text { in } L^{2}(W \cap(M \times[2,4]) \\
& H_{N_{t}^{i}} \longrightarrow H_{N_{t}} \text { a.e. }
\end{aligned}
$$

Now, using Proposition 4.2 we see that

$$
\int_{N_{t}^{i}} \phi F(H) \longrightarrow \int_{N_{t}} \phi F(H), \quad \text { a.e. } t \geq 0 .
$$

Now using (4.8) and the dominated convergence theorem we conclude:

$$
\int_{r}^{s} \int_{N_{t}^{i}} \phi F(H) \longrightarrow \int_{r}^{s} \int_{N_{t}} \phi F(H)
$$

for any $0 \leq r<s$. Note that this same argument will work to prove convergence of the term $\frac{H F(H)}{f(H)}$, as for $\alpha(0)=1$ this term appears as $H^{2}$, and we have shown $L^{2}$ convergence. Now let us consider the term $\frac{F(H)}{f(H)} \nabla \phi \cdot \nu$. Using our uniform bound on $H$ and the area of integration, we clearly have

$$
\int_{N_{t}^{i}} \frac{F(H)}{f(H)}|\nabla \phi \cdot \nu| \leq C(T) \sup _{N_{t}^{i}}|\nabla \phi \cdot \nu| \longrightarrow 0, \quad \text { a.e. } t \geq 0 .
$$

Note that again we have used the fact that for almost all $t, N_{t}^{i}$ converges locally in $C^{1}$ to the vertical cylinder $\widetilde{N}_{t}$, where $\nu$ is perpendicular to $\nabla \phi$. Using (4.8) and the dominated convergence theorem, we then get that

$$
\int_{r}^{s} \int_{N_{t}^{i}} \frac{F(H)}{f(H)}|\nabla \phi \cdot \nu| \longrightarrow 0
$$

Now we address the term $\int_{N_{t}^{i}} \frac{|D f(H)|^{2}}{f(H)^{2}}$. We note that the properties of $f$ and the argument of Lemma 5.1 of [8] show that $f(H)>0 \mathcal{H}^{n-1}$-a.e. on $N_{t}$, so that the integral makes sense for almost all $t$. 
Lemma 4.3 (Lower semicontinuity lemma). For each $0 \leq r<s$,

$$
\int_{r}^{s} \int_{\widetilde{N}_{t}} \phi \frac{|D f(H)|^{2}}{f(H)^{2}} \leq \liminf _{i \rightarrow \infty} \int_{r}^{s} \int_{N_{t}^{i}} \phi \frac{|D f(H)|^{2}}{f(H)^{2}} .
$$

Proof. By (4.11), for a.e. $t \geq 0$ there is a subsequence $i_{j}$ such that

$$
\sup _{j} \int_{N_{t}^{i_{j}} \cap(M \times[2,4])} \frac{|D f(H)|^{2}}{f(H)^{2}}<\infty .
$$

Now let $\hat{N}$ be a connected component of $\tilde{N} \cap(M \times[2,4])$ and let $\hat{N}^{j}$ be a connected component of $N_{t}^{i_{j}} \cap(M \times[2,4])$ converging locally in $C^{1}$ to $\hat{N}$. Let $a_{j}$ be the median of $\log f(H)$ on $\hat{N}_{j}$. By (4.20) and the Rellich compactness theorem, after passing to a further subsequence, there exists $a \in[-\infty, \infty), f \in L^{2}(\hat{N})$ such that $a_{j} \rightarrow a$ and

$$
\log f\left(H_{\hat{N}^{j}}\right)-a_{j} \longrightarrow f \quad \text { in } \quad L^{2}(W) \quad \text { and a.e. on } W
$$

where we have written the surfaces $\hat{N}^{j}$ and $\hat{N}$ as $C^{1}$ graphs over a common surface $W$ for large enough $j$. The case $a=-\infty$ is ruled out for a.e. $t \geq 0$ by the positivity of $H$. If $a>-\infty$, then

$$
\log f\left(H_{\hat{N}^{j}}\right)-a_{j} \longrightarrow \log f\left(H_{\hat{N}}\right)-a \text { in } L^{2}(W) .
$$

Weak convergence in $H_{1}^{2}$ then follows; thus, by the usual lower semicontinuity we get the result for a.e. $t \geq 0$, and hence the desired result by Fatou's lemma.

For the term $D \phi \cdot \frac{D f(H)}{f(H)}$ we note that one can show that this term converges weakly to zero as in Lemma 5.3 of [8] by writing $\int_{N_{t}^{i}} D \phi \cdot \frac{D f(H)}{f(H)}$ as a time derivative, this time using:

$$
\begin{aligned}
g_{i}(t) & :=\int_{N_{t}^{i}}-\epsilon_{i} \phi(z) \frac{\partial}{\partial z} \cdot \frac{D f(H)}{f(H)} d \mu_{N_{t}^{i}}(x, z) \\
& =\int_{N_{t}^{i}}-\epsilon_{i} \phi\left(z-t / \epsilon_{i}\right) \frac{\partial}{\partial z} \cdot \frac{D f(H)}{f(H)} d \mu_{N_{0}^{\epsilon_{i}}}(x, z) .
\end{aligned}
$$

This proves the lemma.

Lemma 4.4. For each $0 \leq r<s$,

$$
\int_{r}^{s} \int_{N_{t}^{i}} D \phi \cdot \frac{D f(H)}{f(H)} \longrightarrow 0
$$


As for the convergence of the term $|A|^{2}$, we will need to define the second fundamental form of a surface in $H_{2}^{2} \cap C^{1}$. We refer the reader to page 402 of [8] for a complete discussion, and here merely state that a weak definition exists, and given $N^{i} \rightarrow N$ locally in $C^{1}$ with

$$
\sup _{i} \int_{N^{i}}\left|A_{N^{i}}\right|^{2}<\infty
$$

then $A_{N}$ exists in $L^{2}(N)$ with weak $L^{2}$ convergence and lower semicontinuity

$$
\int_{N}\left|A_{N}\right|^{2} \leq \liminf _{i \rightarrow \infty} \int_{N^{i}}\left|A_{N^{i}}\right|^{2} .
$$

If we further assume that $\int_{N}|A|^{2}<\infty$ then we may approximate strongly in $C^{1}$ and $H_{2}^{2}$ by smooth surfaces $N^{i}$. These facts are used to prove Lemma 5.4 of $[8]$.

Lemma 4.5 (Weak Gauss-Bonnet formula). Suppose $N$ is a compact $C^{1}$ surface in a 3-manifold, satisfying $\int_{N}|A|^{2}<\infty$. Then

$$
\int_{N} K_{12}+\lambda_{1} \lambda_{2}=2 \pi \chi(N)
$$

where $K_{12}$ denotes the sectional curvature in the ambient manifold evaluated on $T_{x}(N)$ and $\lambda_{i}$ are the eigenvalues of $A$.

Lemma 4.6. Let $N_{t}$ be the limiting surfaces defined above. Then

$$
\int_{N_{t}}|A|^{2} \leq C(T), \quad 0 \leq t \leq T
$$

Proof. By (4.11) and (4.21), we have

$$
\int_{N_{t}}|A|^{2} \leq \infty \quad \text { for a.e. } t \geq 0
$$

Since the surfaces $N_{t}$ are connected and compact with locally uniform $C^{1}$ estimates we have bounds on $\chi\left(N_{t}\right),\left|N_{t}\right|$ and $\sup _{N_{t}}\left|K_{12}\right|$ on $0 \leq t \leq T$. 
Lemma 4.5 gives

$$
\int_{N_{t}} \lambda_{1} \lambda_{2} \leq C(T) \quad \text { for a.e. } t \in[0, T]
$$

Since $H=\lambda_{1}+\lambda_{2}$ is also bounded we get the required bound for almost all $t$. By choosing a sequence of times for which the result holds and applying (4.21) we get the result for all times.

We will also need Lemma 5.6 of [8].

Lemma 4.7. Suppose $E$ is precompact, $E^{\prime}=E$, and $\partial E$ is $C^{1,1}$. Then either $\partial E$ is a smooth minimal surface, or $\partial E$ can be approximated in $C^{1}$ from the inside by smooth sets of the form $\partial E_{\tau}$ with $H>0, E_{\tau}^{\prime}=E_{\tau}$ and

$$
\sup _{\tau} \sup _{\partial E_{\tau}}|A|<\infty, \quad \int_{\partial E_{\tau}} H^{2} \longrightarrow \int_{\partial E} H^{2} \text { as } \tau \longrightarrow 0
$$

We are now in a position to prove the following proposition. The proof will consist of passing to limits and using all of the previous lemmas to conclude convergence.

Proposition 4.8 (Energy growth formula). Let $M$ be a 3-manifold, $E_{0}$ a precompact open set with $C^{1}$ boundary satisfying

$$
\int_{\partial E_{0}}|A|^{2}<\infty
$$

and $\left(E_{t}\right)_{t>0}$ a family of open sets solving (2.4) with initial condition $E_{0}$. Then for each $0 \leq r<s$,

$$
\begin{aligned}
\int_{N_{r}} F(H) \geq & \int_{N_{s}} F(H) \\
& +\int_{r}^{s} \int_{N_{t}}\left(2 \frac{|D f(H)|^{2}}{f(H)^{2}}+|A|^{2}+\operatorname{Rc}(\nu, \nu)-\frac{H F(H)}{f(H)}\right) \\
= & \int_{N_{s}} F(H)+\int_{r}^{s}-4 \pi \chi\left(N_{t}\right) \\
& +\int_{r}^{s} \int_{N_{t}}\left(2 \frac{|D f(H)|^{2}}{f(H)^{2}}+\frac{1}{2}\left(\lambda_{1}-\lambda_{2}\right)^{2}+R+\frac{1}{2} F(H)\right) .
\end{aligned}
$$

Proof. Some of the arguments used here are identical to those in Theorem 5.7 of [8]; so, we sketch them and refer the reader for more details. 
(1) First of all we assume that $\partial E_{0}$ is smooth and $M$ has a smooth subsolution at infinity. Combining together Lemmas 4.3, 4.4, (4.21), (4.18) and (4.19) we can pass (4.3) to the limit as an inequality, with the limiting cylinders $\widetilde{N}_{t}$ replacing $N_{t}^{\epsilon}$ for a.e. $0<r<s$. Using the fact that $\widetilde{N}_{t}$ is a cylinder for a.e. $t$, every quantity $Q(x, z)=Q(x)$ breaks up as

$$
\int_{\widetilde{N}_{t}} \phi Q d \mu_{\widetilde{N}_{t}}=\int_{2}^{4} \phi d z \int_{N_{t}} Q d \mu_{N_{t}}=\int_{N_{t}} Q d \mu_{N_{t}}
$$

thus we obtain the first inequality in (4.24). To establish the second inequality we note that, using the Gauss equation to rewrite $\operatorname{Rc}(\nu, \nu)$ and the elementary formula $|A|^{2}=\frac{1}{2} H^{2}+\frac{1}{2}\left(\lambda_{1}-\lambda_{2}\right)^{2}$ we see:

$$
\begin{aligned}
2|A|^{2} & +2 \operatorname{Rc}(\nu, \nu)-2 \frac{H F(H)}{f(H)} \\
& =H^{2}+\left(\lambda_{1}-\lambda_{2}\right)^{2}+R-2 K+H^{2}-|A|^{2}-2 \frac{H F(H)}{f(H)} \\
& =R-2 K+\frac{1}{2}\left(\lambda_{1}-\lambda_{2}\right)^{2}+\frac{3}{2} H^{2}-2 \frac{H F(H)}{f(h)} \\
& =R-2 K+\frac{1}{2}\left(\lambda_{1}-\lambda_{2}\right)^{2}-F(H)
\end{aligned}
$$

where in the last line we have used the defining differential equation for $F$. Applying Lemmas 4.5 and 4.6 the result follows.

(2) Now we must show that (4.24) holds at $r=0$. From Theorem 2.7 (part (3)) and (2.10), we know that $\partial E_{0}^{\prime}$ is $C^{1,1}$ with $H \geq 0$ and

$$
\int_{\partial E_{0}^{\prime}} F(H) \leq \int_{\partial E_{0}} F(H)
$$

Thus it suffices to show (4.24) for $E_{0}^{\prime}$. We will break into two cases. First say that $H>0$ somewhere on $\partial E_{0}^{\prime}$. Then by Lemma 4.7 there exists a family of smooth surfaces of the form $\partial E_{\tau}$ approximating $\partial E_{0}$ in $C^{1}$, with $H>0$ and $E_{\tau}^{\prime}=E_{\tau}$. By Theorem 3.2, there is a proper solution $\left(E_{t}^{\tau}\right)_{t>0}$ with initial condition $E_{0}^{\tau}=E_{\tau}$. By the smooth existence lemma, we have a smooth flow for short time, thus (4.24) holds for $\left(E_{t}^{\tau}\right)_{t \geq 0}$ at $r=0$, and we just need to pass to the limit now. By Theorem 2.12 (part (3)), (4.22) and (3.4) it is clear that we have $C^{1}$ convergence of the different level sets in $\tau$ as $\tau \rightarrow 0$. Note that the arguments given lemma to show convergence of the different terms in (4.24) not involving derivatives of the cutoff function hold 
for general sequences of exact solutions in a 3-manifold. Finally by (4.22),

$$
\int_{\partial E_{\tau}} H^{2} \longrightarrow \int_{\partial E_{0}^{\prime}} H^{2},
$$

thus by Proposition 4.2

$$
\int_{\partial E_{\tau}} F(H) \longrightarrow \int_{\partial E_{0}^{\prime}} F(H) .
$$

This finishes the proof of (4.24) at $r=0$ in the case where $H>0$ somewhere.

Now suppose that $\partial E_{0}^{\prime}$ is a smooth minimal surface. We make an argument very similar to the previous one where instead of approximating the surface by approximators with positive mean curvature, we approximate the ambient metric by one in which $\partial E_{0}$ has positive mean curvature.

(3) Similarly, when $\partial E_{0}$ is $C^{1}$ satisfying (4.23) we may approximate it by smooth surfaces $S_{i} \subset M-E_{0}$ with $L_{2}$ convergence of the mean curvature by the remark following (4.21). By Theorem 2.12 (part (2)), we have uniform convergence of the solution functions $u_{i} \rightarrow u$. Now, Theorem 2.7 implies that we in fact have $C^{1}$ convergence $\partial E_{t}^{i} \rightarrow N_{t}$ for almost all times. By step (2), (4.24) holds for the approximators and our same arguments allow us to pass to limits assuming $M$ has a smooth subsolution at infinity.

(4) Now we need to remove the assumption of the smooth subsolution at infinity. We do the same conic modification near the asymptotic edge used in the proof of Theorem 3.2 to show the result at $r=0$ under the general hypotheses. By Lemma 4.6, we can apply the result at any $r \geq 0$ to show (4.24) for all times.

Now we recall the result of Corollary 2.10, which is the last step needed to prove the monotonicity.

Proposition 4.9 (Generalized Geroch monotonicity). Let $M$ be a complete 3-manifold, $E_{0}$ a precompact open set with $C^{1}$ boundary satisfying (4.23) and $\left(E_{t}\right)_{t>0}$ a solution of (2.4) with initial condition $E_{0}$. If $E_{0}$ is a minimizing hull then

$$
\begin{aligned}
m_{c}^{F}\left(N_{s}\right) \geq & m_{c}^{F}\left(N_{r}\right)+\frac{1}{(16 \pi)^{3 / 2}} \int_{r}^{s}\left|N_{t}\right|^{1 / 2}\left[16 \pi-8 \pi \chi\left(N_{t}\right)\right. \\
& \left.+\int_{N_{t}} 2|D \log f(H)|^{2}+\left(\lambda_{1}-\lambda_{2}\right)^{2}+R d \mu_{t}\right] d t
\end{aligned}
$$

for $0 \leq r<s$ provided $E_{s}$ is precompact. 


\section{Asymptotic regime}

In this section, we show that $N_{t}$ becomes $C^{1, \alpha}$ close to a coordinate sphere as $t \rightarrow \infty$ by using a blowdown argument and the characterization of solutions to IMCF with compact level sets on $\mathbb{R}^{n}-\{0\}$ proved in [8]. We then show that $m_{\mathrm{ADM}} \geq \lim _{t \rightarrow \infty} m_{c}^{F}\left(N_{t}\right)$ by using an asymptotic expansion of $m_{c}^{F}$. This will complete the proof of Theorem 1.1. As it turns out that the $-\int_{\Sigma} c H^{3}$ in the asymptotic expansion will cancel with the constant $c$ and all of the higher order terms will go to zero, making the analysis from this point in the proof is identical to the case of usual IMCF.

Now let $\Omega$ be the asymptotically flat end of $M$ embedded in $\mathbb{R}^{n}$. Let $g$ be the metric of $M$ pulled back to $\Omega$ with connection $\nabla$, and let $\delta$ be the Euclidean metric with connection $\bar{\nabla}$. We will write $B_{r}(x)$ for metric balls with respect to $g$, and $D_{r}(x)$ for metric balls with respect to $\delta$. Let $u$ be a solution of (2.4) in $\Omega$, and set $E^{t}:=\{u<t\} \subset \Omega$.

Fix $\lambda>0$ and define blown-down objects

$$
\Omega^{\lambda}:=\lambda \cdot \Omega, g^{\lambda}(x):=\lambda^{2} g(x / \lambda), u^{\lambda}(x):=u(x / \lambda), E_{t}^{\lambda}:=\lambda \cdot E_{t} .
$$

Note now that for our generalized flow, the blowndown objects do not satisfy (2.4) because of the non-homogeneity of $F$. However, the gradient estimate together with the fact that our flow approaches the usual IMCF as $\nabla u \rightarrow$ 0 will show that in fact the blowndown limit exists and that the limit is the expanding sphere solution for IMCF. In fact, the blowndown functions satisfy

$$
\lambda C_{F}^{1}\left(|\nabla u|^{2}\right) \leq \operatorname{div}_{M} \frac{\nabla u}{|\nabla u|}-|\nabla u| \leq \lambda C_{F}^{2}\left(|\nabla u|^{2}\right) .
$$

Lemma 5.1 (Blowdown lemma). Suppose the flat metric on $\Omega$ satisfies

$$
|g-\delta|=o(1), \quad|\bar{\nabla} g|=o\left(\frac{1}{|x|}\right)
$$

as $|x| \rightarrow \infty$. Let $u$ be a solution of (2.4) on $\Omega$ such that $\{u=t\}$ is compact for sufficiently large $t$. Then for some constants $c_{\lambda} \rightarrow \infty$,

$$
u^{\lambda}-c_{\lambda} \longrightarrow(n-1) \log |x| .
$$

Proof. We begin with the eccentricity estimates. Fix $t_{0}$ such that $\{u=t\}$ is compact for all $t \geq t_{0}$. By our assumption of asymptotic flatness there is 
$R_{0}>0$ such that

$$
\sigma(x) \geq c|x|, \quad \operatorname{dist}\left(x, \partial E_{t_{0}}\right) \geq c|x| .
$$

Also by the gradient estimate in the existence theorem we have

$$
|\nabla u(x)| \leq \frac{C}{|x|}, \quad \text { for all }|x| \geq R_{0} .
$$

Let $\Theta(N)$ denote the eccentricity of $N$. Recall the definition of $\Theta(N)$ : if $[r(N), R(N)]$ is the smallest interval such that $N \subset D_{R} \backslash D_{r}$, we let $\Theta(N)=$ $R(N) / r(N)$. Using (5.2) and the fact that $f^{\prime}(0)>0$ we see that there exists $A>0$ and $t_{1}$ such that $\left(D_{e^{A t}}\right)_{t_{1} \leq t<\infty}$ is a subsolution of (2.4). Using this surface for comparison we see that

$$
R\left(N_{t+\theta}\right) \leq e^{A \theta} R\left(N_{t}\right), \quad t \geq t_{1}, \quad \theta \geq 0 .
$$

Now we already know $r=r\left(N_{t}\right) \geq R_{0}$. Now $u=t$ somewhere on $\partial D_{r}$, thus by (5.3), there is $C_{2}$ such that $u>t-C_{2}$ everywhere on $\partial D_{r}$. Thus, $N_{t-C_{2}} \cap$ $\partial D_{r}=\phi$. By Lemma 4.1, $N_{t-C_{2}}$ cannot have any components outside of $D_{r}$, thus $R\left(N_{t-C_{2}}\right) \leq r$. Combining this with (5.4) gives

$$
R\left(N_{t}\right) \leq e^{A C_{2}} R_{N_{t-C_{2}}} \leq e^{A C_{2}} r\left(N_{t}\right) .
$$

We must now show that a limit solution exists. Let $\lambda_{i} \rightarrow 0$. Note that the estimates (5.3), (5.4) and (5.5) are scale-invariant, and thus hold for $N_{t}^{\lambda_{i}}$ on the complement of a subset shrinking to $\{0\}$ as $i \rightarrow \infty$. Using these estimates and Theorem 2.7 we can modify the proof of Theorem 2.11 to show that there exists a subsequence $\left(\lambda_{i_{j}}\right)$ and numbers $c_{j} \rightarrow \infty$ and a limit function $v$ such that $u^{\lambda_{i_{j}}}-c_{j} \rightarrow v$ locally uniformly in $\mathbb{R}^{n} \backslash\{0\}$ with local $C^{1}$ convergence of the level sets. It is clear now using (5.1) that $v$ satisfies the usual IMCF equation. Equation (5.3) shows that $v$ is not constant, making some level set non-empty. The bounded eccentricity combined with the fact that all large enough level sets are compact shows that in fact the level sets of $v$ are all compact. Thus, applying Proposition 7.2 of [8] the result follows.

We now recall the definition of ADM mass. The proof of part 1 is found in [1], of part (2) is found in [3] and [6].

Lemma 5.2 (ADM lemma). Suppose $R \geq 0$ on $M$, and the asymptotic region $\Omega$ is embedded as the complement of a compact set in $\mathbb{R}^{3}$ equipped with 
the flat metric $\delta$. Let $U$ be a precompact open set with smooth boundary, $\nu$ the outward unit normal of $U$ with respect to $g$, and $d \mu$ the surface measure of $\partial U$ with respect to $g$.

1. If $\delta$ satisfies

$$
c \delta \leq g \leq C \delta \quad \text { in } \Omega, \quad \int_{\Omega}|\bar{\nabla} g|^{2}<\infty
$$

then the limit

$$
m_{\mathrm{ADM}}(g, \delta):=\lim _{U \rightarrow M} \frac{1}{16 \pi} \int_{\partial U} g^{i j}\left(\bar{\nabla}_{j} g_{i k}-\bar{\nabla}_{k} g_{i j}\right) \nu^{k} d \mu
$$

exists and is finite if and only if $\int_{M} R<\infty$. Here $\chi_{U} \rightarrow \chi_{M}$ locally uniformly.

2. If $\delta$ satisfies

$$
|g-\delta| \leq C|x|^{-1 / 2-\alpha}, \quad|\bar{\nabla} g| \leq C|x|^{-3 / 2-\alpha}, \quad x \in \Omega
$$

for some $\alpha>0$, then $m_{\mathrm{ADM}}$ is a geometric invariant of $g$, independent of the choice of $\delta$.

Lemma 5.3 (Asymptotic comparison lemma). Assume that $M$ is asymptotically flat, and let $\left(E_{t}\right)_{t \geq t_{0}}$ be a family of precompact subsets weakly solving (2.4) in $M$. Then

$$
\lim _{t \rightarrow \infty} m_{c}^{F}\left(N_{t}\right) \leq m_{A D M}(M)
$$

Proof. Define $r=r(t)$ by $\left|N_{t}\right|=4 \pi r^{2}$. Then $\left|N_{t}^{1 / r}\right|_{g^{1 / r}}=4 \pi$. Thus Lemma 5.1 implies that

$$
N_{t}^{1 / r(t)} \longrightarrow \partial D_{1} \quad \text { in } C^{1} \text { as } t \longrightarrow \infty
$$

Let $h$ be the restriction of $g$ to the surface $N_{t}$ and let $\epsilon$ be the restriction of the flat metric to it. Let $\eta$ denote the exterior unit normal, $\omega$ the unit dual normal, $A$ the second fundamental form and $H$ the mean curvature, all with respect to $g$. The quantities $\bar{\eta}$ and others are the corresponding quantities with respect to the flat metric. Now, let $p=g-\delta$. We restrict to the region where $|p| \leq \frac{1}{10}$ on $N_{t}$. We record some basic estimates for 
geometric quantities in an asymptotically flat manifold. First of all

$$
\begin{aligned}
\Gamma_{i j}^{k} & =\frac{1}{2} g^{k l}\left(\nabla_{i} p_{j l}+\nabla_{j} p_{i l}-\nabla_{l} p_{i j}\right) \pm C|p||\nabla p| \\
\nabla p & =\bar{\nabla} p \pm C|p||\nabla p| .
\end{aligned}
$$

Also the following estimates are derived in page 418 of [8]:

$$
\begin{aligned}
|H-\bar{H}| \leq & C|p||A|+C|\nabla p|, \quad\left|H^{2}-\bar{H}^{2}\right| \leq C|p||A|^{2} \\
& +C|\nabla p|+C|\nabla p||A| \\
\bar{H}^{2}(d \mu-d \bar{\mu})= & \left(\frac{1}{2} H^{2} h^{i j} p_{i j} \pm C|p|^{2}|A|^{2} \pm C|\nabla p|^{2}\right) d \mu .
\end{aligned}
$$

Note also that we know

$$
\int_{N} \bar{H}^{2} d \bar{\mu} \geq 16 \pi
$$

thus we have

$$
\begin{aligned}
\int_{N_{t}} F(H) d \mu= & \int_{N_{t}} H^{2} d \mu-\int_{N_{t}} c H^{3} d \mu+\int_{N_{t}} \mathcal{O}\left(H^{4}\right) d \mu \\
\geq & \int_{N_{t}} \bar{H}^{2} d \bar{\mu}+\bar{H}^{2}(d \mu-d \bar{\mu})+2 H(H-\bar{H})-(H-\bar{H})^{2} d \mu \\
& -\int_{N_{t}} c H^{3} d \mu+\int_{N_{t}} \mathcal{O}\left(H^{4}\right) d \mu \\
\geq & 16 \pi-\int_{N_{t}} c H^{3}+\int_{N_{t}} \mathcal{O}\left(H^{4}\right)+\int_{N_{t}} \frac{1}{2} H^{2} h^{i j} p_{i j} \\
& -2 H h^{i k} p_{k l} h^{l j} A_{i j}+H^{2} \nu^{i} \nu^{j} p_{i j}-2 H h^{i j} \nu^{l} \nabla_{i} p_{j l} \\
& +H h^{i j} \nu^{l} \nabla_{l} p_{i j}-C|p|^{2}|A|^{2}-C|\nabla p|^{2} .
\end{aligned}
$$

Now we will show that $m_{F}\left(N_{t}\right)$ remains bounded. Recall that we have the following estimate

$$
|H|=f^{-1}(|\nabla u|) \leq \frac{C}{|x|} \leq \frac{C}{r} \quad \text { on } \quad N_{t}
$$


for a.e. sufficiently large $t$. Using this with our growth formula,

$$
\begin{aligned}
\int_{s}^{s+1} \int_{N_{t}}|A|^{2} & =\int_{s}^{s+1} \int_{N_{t}} \frac{1}{2}\left(\lambda_{1}-\lambda_{2}\right)^{2}+\frac{1}{2} H^{2} \\
& \leq \int_{N_{s}} F(H)+\int_{s}^{s+1} 4 \pi \chi\left(N_{t}\right)+\int_{s}^{s+1} \frac{1}{2} H^{2} \\
& \leq 4 \pi C_{F}+8 \pi+4 \pi C .
\end{aligned}
$$

Thus, we may select a subsequence $t_{i} \rightarrow \infty$ such that

$$
\sup _{i} \int_{N_{t_{i}}}|A|^{2}<\infty
$$

Note also by (5.9) that

$$
\int_{N_{t}} \mathcal{O}\left(H^{3}\right)=\frac{C}{r}
$$

Together with (5.8), we then have

$$
\int_{N_{t_{i}}} F(H) \geq 16 \pi-\frac{C}{r} .
$$

Thus by the definition and monotonicity of $m_{F}\left(N_{t}\right)$, we have

$$
\sup _{t} m_{F}\left(N_{t}\right)<\infty
$$

Now, using the monotonicity formula, we see that

$$
\int_{t_{0}}^{\infty} e^{c_{F} t} \int_{N_{t}}\left(\lambda_{1}-\lambda_{2}\right)^{2}+\frac{|D f(H)|^{2}}{f(H)^{2}}<\infty .
$$

Thus, there exists a subsequence $t_{j} \rightarrow \infty$ such that

$$
\int_{N_{j}}\left(\lambda_{1}-\lambda_{2}\right)^{2}+\frac{|D f(H)|^{2}}{f(H)^{2}} \longrightarrow 0 .
$$

Given our conditions on $F$ and the bound $H \leq C / r$ it is clear that this implies an $H_{1}^{2}$ bound on $H$. Considering the rescaled surfaces $N_{j}^{1 / r_{j}}$ as graphs over the disc for large enough $j$, it is clear that we can apply Rellich 
compactness to conclude

$$
H_{N_{j}^{1 / r_{j}}} \longrightarrow H_{\partial D_{1}}=2 \text { in } L^{2}\left(\partial D_{1}\right) .
$$

This then implies that

$$
H_{N_{j}}=\frac{2}{r}+f_{j} \text { on } N_{j}
$$

where $\int_{N_{j}} f_{j}^{2} \rightarrow 0$. Also, we have

$$
\int_{N_{j}}\left|A-\frac{H}{2} h\right|^{2}=\frac{1}{2} \int-N_{j}\left(\lambda_{1}-\lambda_{2}\right)^{2} \longrightarrow 0
$$

thus

$$
A_{N_{j}}=\frac{h}{r}+g_{j} \quad \text { on } N_{j}
$$

where $\int g_{j}^{2} \rightarrow 0$. Thus

$$
\sup _{j} \int_{N_{j}}|A|^{2}<\infty
$$

Finally, we note by Lemma 5.1 that in fact $H=\frac{2}{r}+\mathcal{O}\left(\frac{1}{r^{2}}\right)$ so that

$$
r_{l} \int_{N_{t}} c H^{3}=32 \pi c+\frac{C}{r} .
$$

We now can estimate

$$
\begin{aligned}
32 \pi m_{c}\left(N_{l}\right)= & r_{l}\left(16 \pi-\int_{N_{l}} F(H)\right)-32 \pi c \\
= & r_{l}\left(16 \pi-\int_{N_{t}} H^{2} d \mu+\int_{N_{t}} c H^{3} d \mu+\int_{N_{t}} \mathcal{O}\left(H^{4}\right) d \mu\right)-32 \pi c \\
= & r_{l}\left(16 \pi-\int_{N_{t}} H^{2} d \mu\right)+\frac{C}{r} \\
\leq & \frac{C}{r_{l}}+r_{l} \int_{N_{l}}-\frac{1}{2} H^{2} h^{i j} p_{i j}+2 H h^{i k} p_{k l} h^{l j} A_{i j} \\
& -H^{2} \nu^{i} \nu^{j} p_{i j}+2 H h^{i j} \nu^{l} \nabla_{i} p_{j l}-H h^{i j} \nu^{l} \nabla_{l} p_{i j} \\
\leq & \frac{C}{r_{l}}+\eta_{l}+\int_{N_{l}}-\frac{2}{r_{l}} h^{i j} p_{i j}+\frac{4}{r_{l}} h^{i j} p_{i j}-\frac{4}{r_{l}} \nu^{i} \nu^{j} p_{i j} \\
& +4 h^{i j} \nu^{l} \nabla_{i} p_{j l}-2 h^{i j} \nu^{l} \nabla_{l} p_{i j}
\end{aligned}
$$


where $\eta_{l}$ is an error term that goes to zero in the limit because of the condition of asymptotic flatness (5.2). We can integrate by parts to see that

$$
\begin{aligned}
\int_{N_{l}} h^{i j} \nu^{l} \nabla_{i} p_{j l} & =\int_{N_{l}} H \nu^{j} \nu^{l} p_{j l}-h^{i j} p_{j k} h^{k l} A_{l i} \\
& =\int_{N_{l}} \frac{2}{r_{l}} \nu^{j} \nu^{l} p_{j l}-h^{i j} p_{i j}+\eta_{l}^{\prime}
\end{aligned}
$$

where $\eta_{l}^{\prime}$ goes to zero in the limit by (5.2). Using this in the previous equation gives

$$
32 \pi m_{c}\left(N_{l}\right) \leq \frac{C}{r_{l}}+\eta_{l}+2 \eta_{l}^{\prime}+\int_{N_{l}} 2 h^{i j} \nu^{l} \nabla_{i} p_{j l}-2 h^{i j} \nu^{l} \nabla_{l} p_{i j} .
$$

By (5.7), this quantity approaches the ADM mass. Applying the monotonicity formula, we have

$$
\sup _{t \geq 0} m_{c}\left(N_{t}\right) \leq m_{\mathrm{ADM}}(M)
$$

\section{Acknowledgements}

The author would like to thank Hubert Bray for suggesting this problem and for many helpful discussions throughout the preparation of this paper. He would also like to thank the referee for helpful comments in improving the exposition.

\section{References}

[1] R. Arnowitt, S. Deser and C.W. Misner, Coordinate invariance and energy expressions in general relativity, Phys. Rev. 122 (2), 1961, 997-1006.

[2] T. Aubin, Some nonlinear problems in Riemannian geometry, Springer Monographs in Mathematics, Springer-Verlag, Berlin, 1998.

[3] R. Bartnik, The mass of an asymptotically flat manifold, Comm. Pure Appl. Math. 39, 1986, 661-693.

[4] H. Bray, A family of quasi-local mass functionals with monotone flows, Proceedings of the International Congress of Mathematical Physics, Lisbon, 2003. 
[5] - Negative point mass singularities in general relativity, www. newton.cam.ac.uk/webseminars/pg+ws/2005/gmr/0830/bray, 2005.

[6] P. Chruściel, Boundary conditions at spatial infinity from a Hamiltonian point of view, Topological properties and global structure of space-time (Erice, 1985), NATO Adv. Sci. Inst. Ser. B Phys. 138, New York, 1986.

[7] D. Gilbarg and N.S. Trudinger, Elliptic partial differential equations of second order, Classics in mathematics, Springer-Verlag, Berlin, 2001.

[8] G. Huisken and T. Ilmanen, The inverse mean curvature flow and the Riemannian Penrose inequality, J. Differen. Geom. 59, 2001, 353-437.

[9] N. Robbins, Negative point mass singularities in general relativity, Ph.D. thesis, Duke University, 2007.

[10] J. Streets, Point mass singularities, Preprint, 2008.

Fine HaLL

WASHINGTON RoAD

PRINCETON, NJ 08544

USA

E-mail address: jstreets@math.princeton.edu

Received December 19, 2006 
